ARTICLE

Received 28 Jan 2013 | Accepted 18 Jul 2013 | Published 14 Aug $2013 \quad$ DOl: 10.1038/ncomms3330

\title{
Bcl-wav and the mitochondrial calcium uniporter drive gastrula morphogenesis in zebrafish
}

Julien Prudent', Nikolay Popgeorgiev¹, Benjamin Bonneau', Julien Thibaut ${ }^{1}$, Rudy Gadet ${ }^{1}$, Jonathan Lopez ${ }^{1,2}$, Philippe Gonzalo ${ }^{1,2}$, Ruth Rimokh¹, Stephen Manon ${ }^{3}$, Corinne Houart ${ }^{4}$, Philippe Herbomel ${ }^{5}$, Abdel Aouacheria ${ }^{6}$ \& Germain Gillet ${ }^{1}$

Bcl-2 proteins are acknowledged as key regulators of programmed cell death. However, increasing data suggest additional roles, including regulation of the cell cycle, metabolism and cytoskeletal dynamics. Here we report the discovery and characterization of a new Bcl-2related multidomain apoptosis accelerator, Bcl-wav, found in fish and frogs. Genetic and molecular studies in zebrafish indicate that Bcl-wav and the recently identified mitochondrial calcium uniporter (MCU) contribute to the formation of the notochord axis by controlling blastomere convergence and extension movements during gastrulation. Furthermore, we found that $\mathrm{Bcl}$-wav controls intracellular $\mathrm{Ca}^{2}+$ trafficking by acting on the mitochondrial voltage-dependent anion channel, and possibly on MCU, with direct consequences on actin microfilament dynamics and blastomere migration guidance. Thus, from an evolutionary point of view, the original function of $\mathrm{Bcl}-2$ proteins might have been to contribute in controlling the global positioning system of blastomeres during gastrulation, a critical step in metazoan development.

\footnotetext{
${ }^{1}$ Université de Lyon, Centre de recherche en cancérologie de Lyon, U1052 INSERM, UMS 3453 CNRS, Université Lyon I, Centre Léon Bérard, 28 rue Laennec, Lyon 69008, France. ${ }^{2}$ Hospices Civils de Lyon, Fédération de Biochimie Nord, Hôpital de la Croix-Rousse, 103 Grande rue de la Croix-Rousse, Lyon Cedex 04 F-69317, France. ${ }^{3}$ Université de Bordeaux, Institut de génétique et biochimie cellulaires, UMR 5095 CNRS, 1 rue Camille Saint-Saëns, Bordeaux Cedex 33077 , France. ${ }^{4}$ MRC Centre for Developmental Neurobiology, King's college, University of London, NHH, Guy's Campus, London SE1 1UL, UK. ${ }^{5}$ Département de Biologie du Développement, CNRS-URA 2578, Institut Pasteur, 25 rue du Dr Roux, Paris Cedex 15 75724, France. ${ }^{6}$ Laboratoire de Biologie Moléculaire de la Cellule, Ecole Normale Supérieure de Lyon, UMR 5239 CNRS-UCBL-ENS Lyon, Université de Lyon, SFR BioSciences Gerland, Lyon Sud (UMS3444/US8), 46 Allée d'Italie, Lyon Cedex 07 69364, France. Correspondence and requests for materials should be addressed to G.G. (email: germain.gillet@univ-lyon1.fr).
} 
cl-2 proteins are major regulators of programmed cell death. During early vertebrate development, they shape the embryo body through sustained cell homoeostasis and tissue morphogenesis. At the molecular level, Bcl-2 proteins act by controlling the mitochondrial release of cytotoxic molecules such as cytochrome- $c$, leading to caspase activation and subsequent apoptosis. The permeabilization of the outer mitochondrial membrane (OMM), which represents a key step during apoptosis, is regulated by the insertion of the apoptosis accelerators Bax and Bak and prevented by Bcl-2 and related apoptosis inhibitors 1,2 .

In addition to apoptosis, mitochondria has a central role in intracellular calcium homoeostasis by rapidly transporting $\mathrm{Ca}^{2+}$ from the cytosol to the mitochondrial matrix ${ }^{3,4}$. This process requires the passage of $\mathrm{Ca}^{2+}$ ions through the voltage-dependent anion channel (VDAC) at the $\mathrm{OMM}^{5}$. At the inner mitochondrial membrane, this process is completed via the mitochondrial calcium uniporter (MCU), which was described only recently 6,7 . Members of the Bcl-2 family actively participate in the control of calcium homoeostasis by binding to VDAC and controlling its $\mathrm{Ca}^{2+}$ permeability ${ }^{8}$. Despite these findings, the majority of studies have been performed in vitro, and the physiological relevance of this calcium control at the level of the whole organism, especially during the first steps of vertebrate development, remains unclear.

Zebrafish has become a powerful model organism for studying the role of genes related to early vertebrate development and apoptosis ${ }^{9}$. During this stage, a set of morphogenetic movements known as convergence and extension (C\&E) begins; these movements shape the embryo body in a co-ordinated fashion. $\mathrm{C} \& \mathrm{E}$ movements are characterized by blastomere migration from the ventral to the dorsal region and at the animal pole, resulting in the formation of the anteroposterior and mediolateral axes ${ }^{10}$. Interestingly, during these early stages, $b c l-2$ and related genes are actively transcribed, which suggests their implication in the zebrafish development ${ }^{11}$. Moreover, $\mathrm{Ca}^{2+}$ signalling has a crucial role in early development, particularly during gastrulation ${ }^{12}$.

Recently, we have characterized a Bcl-2-related anti-apoptotic protein, $\mathrm{Nrz}^{13}$. The Nrz protein controls the first morphogenetic movement, known as epiboly, during early zebrafish development ${ }^{14}$. At the molecular level, epiboly is achieved through Nrz-dependent intracellular calcium control, highlighting the emerging role of $\mathrm{Bcl}-2$ proteins as multifunctional cell factors during early vertebrate development.

Here we present the identification of the new apoptosis accelerator Bcl-wav, named for its expression in water-living vertebrates (anamniotes). The Bcl-wav protein shares structural and functional similarities, including its mitochondrial localization, with multidomain death accelerators. Knockdown of bclwav or $m c u$ in zebrafish results in major alterations in the C\&E movements of blastomeres during gastrulation, presumably due to alterations in F-actin dynamics. At later stages, we found that the maturation of the notochord and anteroposterior axis formation were compromised by bclwav or $\mathrm{mcu}$ silencing. At the molecular level, Bcl-wav interacted with both VDAC type 1 (VDAC1) and MCU, demonstrating that it is critical for mitochondrial $\mathrm{Ca}^{2+}$ uptake. Finally, Bcl-wav and MCU controlled the overall blastomere positioning system during gastrulation via the regulation of intracellular calcium trafficking. Together, our data support the notion that the $\mathrm{Bcl}-2$ proteins have key roles in early development by controlling actin microfilament dynamics and thus also cell movement.

\section{Results}

Expression of bclwav during zebrafish development. We recently developed a set of structure-based hidden Markov model profiles specific for cellular and/or viral-encoded proteins that share the same three-dimensional fold as Bcl-2 (ref. 15). These novel models reliably identified Bcl-2 family-related proteins in a vast array of metazoans, including members with low sequence similarity. During the course of these studies, we discovered a previously uncharacterized open-reading frame present in the zebrafish genome, which we believed was likely to encode a new Bcl-2 homologue. Subsequent BLAST searches identified expressed sequence tags (ESTs) with significant similarity to the corresponding putative product in teleosts and anurans; however, no corresponding EST was found in birds or mammals. This sequence was thus named Bcl-wav, for 'water-living anamniote vertebrates' (Fig. 1a). The bclwav sequence can be found in GenBank under accession number GU350411.1 (NCBI NP_001165873). Local alignment matrices showed that the zebrafish and Xenopus Bcl-wav proteins clustered with multidomain Bcl-2 members, consistent with Bcl-wav primary sequence analyses, which identified four Bcl-2 homology $(\mathrm{BH})$ domains and a C-terminal transmembrane (TM) domain (Fig. 1b,c). Moreover, secondary structure predictions revealed an all-alpha helical structure, which is typical of Bcl-2-like proteins (Fig. 1c).

To investigate bclwav expression, we performed reverse transcriptase PCR experiments on zebrafish embryos. Bclwav mRNA could be detected as early as the 128-cell stage, that is, before the mid-blastula transition ${ }^{16}$, indicating that the bclwav transcript is maternally inherited (Fig. 1d). Bclwav expression was further analysed by in situ hybridization using two nonoverlapping bclwav probes and one full-length bclwav probe. The same expression pattern was obtained with all three probes (Fig. 1d). Indeed, bclwav was ubiquitously expressed up to $14 \mathrm{~h}$ post fertilization (hpf), whereas at later stages $(36-48 \mathrm{hpf})$ its expression became somite-specific. Interestingly, these results corroborated the fact that bclwav ESTs were found in muscle tissue (Salmo salar, smus1-014AD07.g1 and Xenopus tropicalis, XP_002940781.1), suggesting a conserved expression pattern across species.

Bcl-wav is a mitochondrial pro-apoptotic Bcl-2 family member. To gain insight into the function of Bcl-wav, we first investigated its subcellular distribution in vitro. Confocal microscopy analyses showed that Bcl-wav was mainly localized in the mitochondria ( $85 \pm 6.8 \%$ co-localization with Mitotracker, $n=5$; measured using Zeiss LSM 780 Zen software); however, the Bcl-wav $\Delta \mathrm{TM}$ mutant, which lacked the C-terminal TM domain, was cytosolic (Fig. 2a,b and Supplementary Fig. S1a,b). Analyses of purified mitochondria from zebrafish embryos injected with in vitrosynthesized mRNAs encoding either full-length Bcl-wav or the $\mathrm{Bcl}-\mathrm{wav} \Delta \mathrm{TM}$ deletion mutant showed that Bcl-wav $\Delta \mathrm{TM}$ was unable to localize in the mitochondrial fraction (Supplementary Fig. S1b). Notably, deleting the BH4 domain did not affect Bcl-wav subcellular localization (Fig. 2b). Furthermore, the Bclwav protein appeared to be tightly anchored to the mitochondria, as demonstrated by an $\mathrm{Na}_{2} \mathrm{CO}_{3}$ treatment assay (Fig. 2c).

Next, we directly evaluated the subcellular localization of the endogenous Bcl-wav protein in zebrafish embryos using a rabbit polyclonal antibody. To this end, mitochondria and endoplasmic reticulum (ER) fractions purified from the yolk syncytial layer (YSL) of embryos at various stages of epiboly were analysed; these experiments showed that Bcl-wav was already present at the sphere stage and gradually accumulated during gastrulation (Fig. 2c,d). Together, these findings showed that Bcl-wav is a resident mitochondrial protein that is presumably inserted into the OMM via its hydrophobic TM domain. The activity of Bclwav was then analysed further. The ectopic expression of Bcl-wav in zebrafish embryos and HeLa cells demonstrated its capacity to 
a

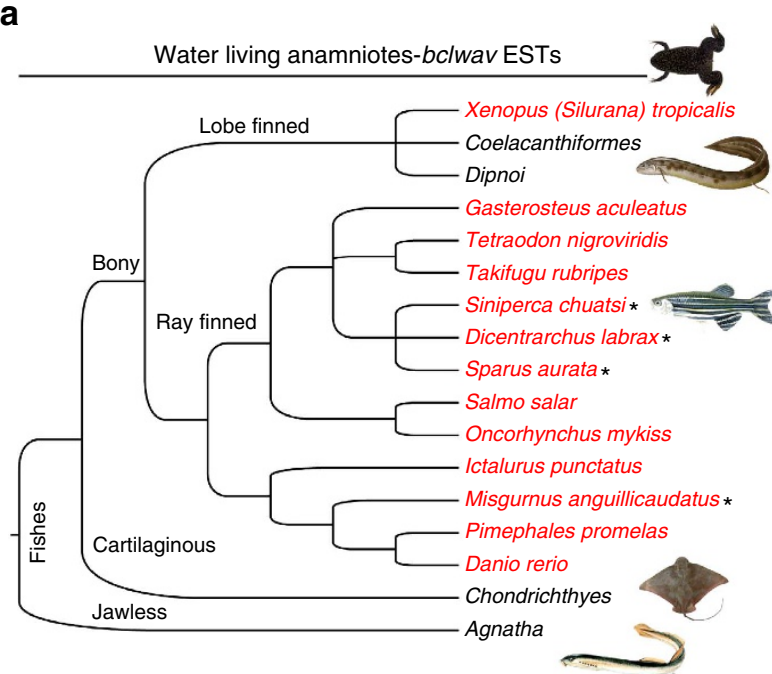

b Bcl-wav Danio Xenopus

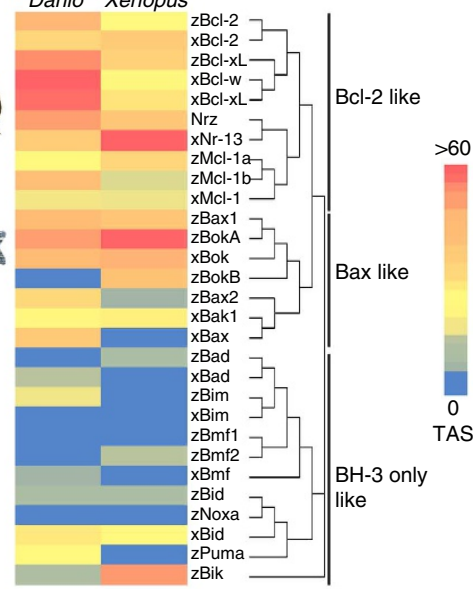

C

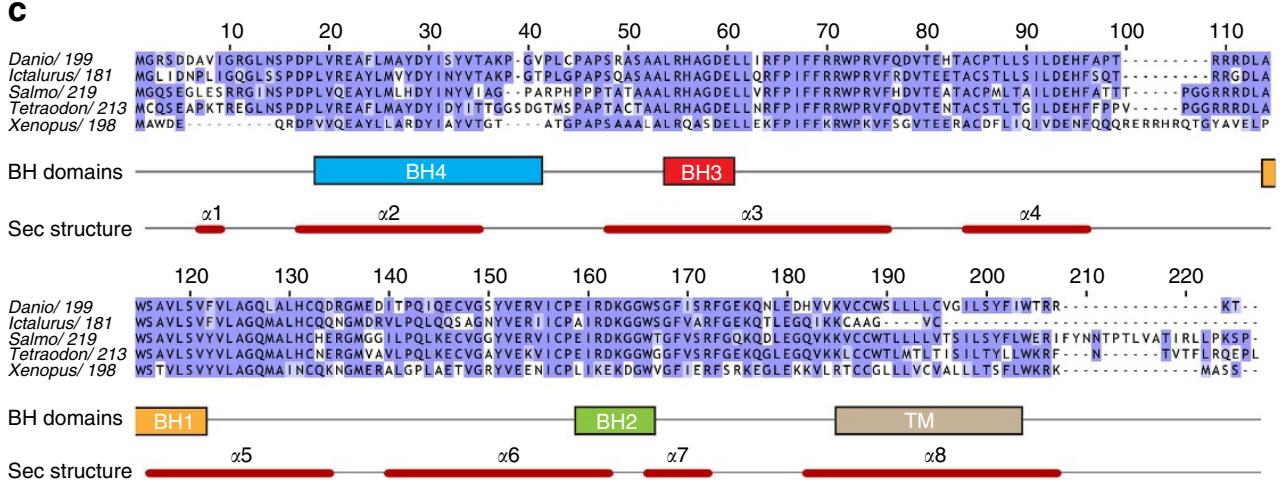

d bclwav expression

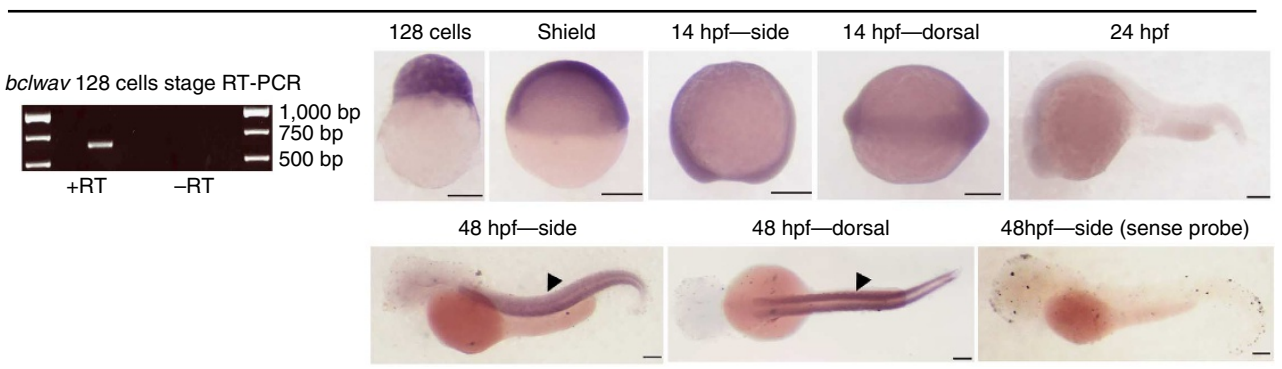

Figure 1 | Sequence and expression analysis of the bclwav gene. (a) Cladogram of bclwav-orthologous ESTs retrieved from GenBank, indicating the restricted phylogenetic distribution of the bclwav gene in aquatic anamniotes. Partial $\left(^{\star}\right)$ and total bclwav ESTs were found in Danio rerio, Pimephales promelas, Misgurnus anguillicaudatus, Oncorhynchus mykiss, Salmo salar, Gasterosteus aculeatus, Tetraodon nigroviridis, Takifugu rubripes, Dicentrarchus labrax, Siniperca chuatsi, Sparus aurata, Ictalurus punctatus and Xenopus tropicalis (red lines). (b) In silico hybridization matrix representing the alignment of $D$. rerio (Bcl-wav) and X. tropicalis (xBcl-wav) bclwav orthologs versus the $\mathrm{Bcl}-2$-related proteins found in these two species. Coloured boxes indicate to the total alignment score (blue, no alignment; red, high alignment score (>60)). Bcl-wav and xBcl-wav cluster mainly with multidomain Bcl-2 members.

(c) ClustalW alignment of Bcl-wav primary structures in multiple species. Identical and similar residues are boxed in blue and cyan, respectively. The positions of conserved $\mathrm{BH} 1-\mathrm{BH} 4$ domains and of the $\mathrm{C}$-terminal TM domain are indicated by coloured boxes below the sequences, and the locations of the predicted alpha helices are indicated by red stripes. (d) Reverse transcriptase PCR of polyA + RNA extracted from whole embryos before the mid-blastula transition (MBT). Bclwav expression was detected in embryos before the MBT ( + RT) (top left panel). A reaction lacking the RT enzyme ( $-\mathrm{RT}$ ) was used as a negative control. Top right and bottom panels show bclwav whole-mount in situ hybridization. Before MBT (128 cells), at the shield (6hpf) and $14 \mathrm{hpf}$ stages, bclwav is ubiquitously expressed. At $24 \mathrm{hpf}$, bclwav expression decreases. In addition, at $48 \mathrm{hpf}$, bclwav is highly expressed in the somites (black arrowheads). A negative control at $48 \mathrm{hpf}$ using a sense probe is shown in the bottom right-outermost panel. Scale bar, $200 \mu \mathrm{m}$.

activate caspase 3 , in contrast to the Bcl-wav $\Delta \mathrm{TM}$ mutant, which failed to do so (Fig. 2e,f and Supplementary Fig. S2a-c). Moreover, caspase activation was prevented by the apoptosis inhibitors zBcl-xL and Nrz (Supplementary Fig. S2b). Notably, the $\triangle \mathrm{BH} 4$ deletion mutant was still found to activate caspase 3 and lead to an increase in cleaved poly(ADP) ribose polymerase
(PARP), a target of caspases during apoptosis, indicating that the $\mathrm{BH} 4$ domain is dispensable for apoptosis induction by $\mathrm{Bcl}-\mathrm{wav}$ (Supplementary Fig. S2c). In addition, Bcl-wav appeared to activate caspase 3 in a Bax/Bak-dependent manner, as shown using bax/bak-deficient mouse embryo fibroblasts (Supplementary Fig. S2d). Interestingly, the Bcl-wav/Bax 

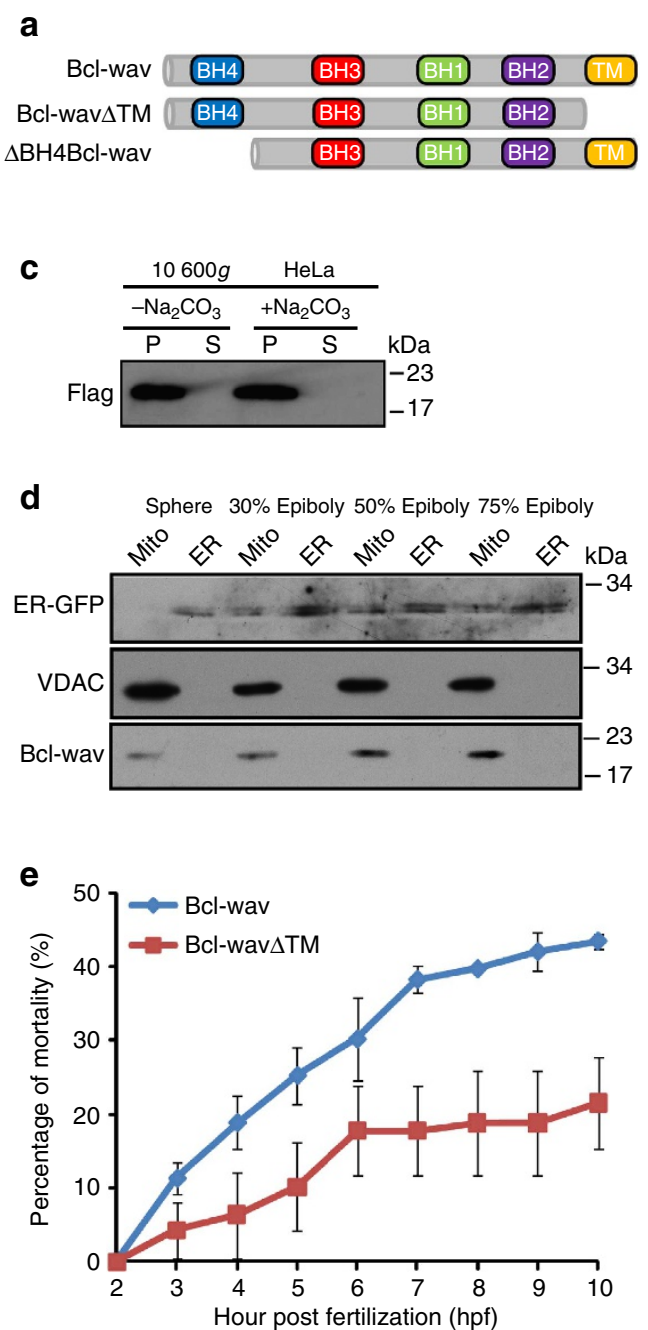

b
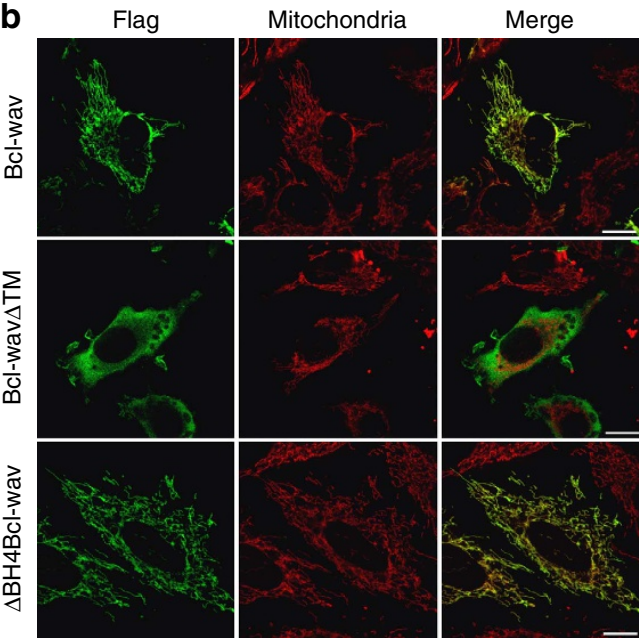

$\mathbf{f}$
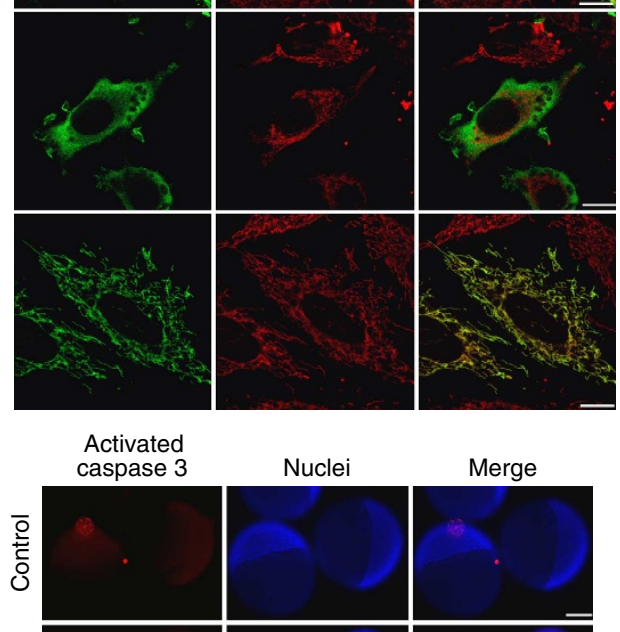

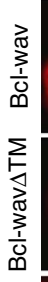
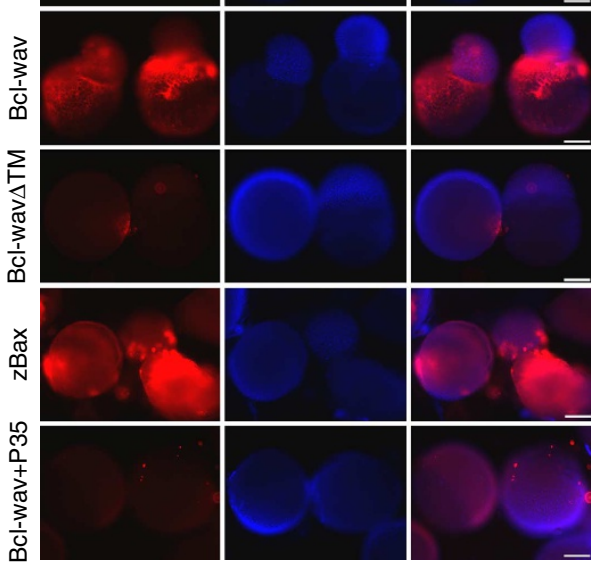

Figure 2 | Bcl-wav is a mitochondrial pro-apoptotic protein. (a) Schematic drawing of recombinant proteins: full-length $B c l-w a v, B c l-w a v \Delta T M$ and $\triangle \mathrm{BH} 4 \mathrm{Bcl}$-wav. The $\mathrm{BH}$ and TM domains are shown as coloured boxes. (b) Analysis of Bcl-wav subcellular localization by confocal microscopy in HeLa cells transiently transfected with pCS2 + Flag-Bcl-wav, pCS2 + Flag-Bcl-wav $\Delta T M$ and pCS2 + Flag- $\Delta \mathrm{BH} 4 \mathrm{Bcl}$-wav expressing Flag-Bcl-wav, Flag-Bcl-wav $\Delta \mathrm{TM}$ and Flag- $\triangle \mathrm{BH} 4 \mathrm{Bcl}$-wav fusion proteins, respectively. Mitochondria were labelled using Mitotracker red dye, and Flag fusion proteins were detected using an anti-Flag antibody. In contrast to Flag-Bcl-wav $\Delta T \mathrm{TM}$, which is localized in the cytosol, Flag-Bcl-wav and Flag- $\Delta \mathrm{BH} 4 \mathrm{Bcl}$-wav co-localize with mitochondria. Scale bar, $5 \mu \mathrm{m}$. (c) Flag-Bcl-wav is inserted into the mitochondrial membrane. Purified mitochondria from Flag-Bcl-wav-expressing cells were treated with sodium carbonate and immunoblotted with the anti-Flag antibody. Flag-Bcl-wav was found in the mitochondrial pellet (P) containing membrane-inserted proteins and was not found in the supernatant (S). (d) Bcl-wav is a mitochondrial zebrafish protein. Subcellular fractionation of YSL mitochondria and YSL endoplasmic reticulum (ER) of embryos expressing ER-GFP at the sphere stage and at 30\%, 50\% and 75\% epiboly. Bcl-wav was detected in the mitochondrial fraction (Mito) and accumulates during gastrulation. VDAC and GFP antibodies were used as mitochondrial and ER markers, respectively. (e) The effect of bclwav overexpression on the mortality of zebrafish embryos. In contrast to bclwav $\Delta T M$ mRNA, bclwav mRNA injection (100 ng/ $\mu$ l) into 1-4 cell stage embryos induces early mortality during gastrulation (mean \pm s.d.; three independent experiments). (f) Bclwav overexpression promotes caspase 3 activation in zebrafish embryos at 50\% epiboly. Left panels: activated caspase 3 staining in embryos injected at the one-cell stage with egfp (control), zbax, bcl-wav $\Delta t m$, bclwav and bclwav plus p35 mRNAs. Embryos were observed at 30-50\% epiboly. Middle panels: the same embryos were stained with Hoechst reagent to visualize the nuclei. Right panels: merged images of stained embryos. Bclwav overexpression induces YSL-specific caspase 3 activation (48\%), which is abrogated by p35 co-injection (8\%). Scale bar, $200 \mu \mathrm{m}$. Full scans of western blots can be found in Supplementary Fig. S9.

interaction was detected by co-immunoprecipitation assays in HeLa cells (Supplementary Fig. S2e). These results were confirmed by experiments in a yeast model, which showed that Bcl-wav could activate Bax and promote cytochrome-c release (Supplementary Fig. S3). Overall, these data suggest that Bcl-wav is a new potential death accelerator that shares structural similarities with multidomain Bcl-2 family members.
Bclwav knockdown leads to major developmental defects. Knockdown experiments with morpholinos were carried out to further investigate Bcl-wav role during development. In these experiments, anti-bclwav morpholinos were co-injected together with an anti- $p 53$ morpholino ( $p 53-\mathrm{MO})$ to abolish the nonspecific induction of $\mathrm{p} 53$-dependent cell death, a frequent off-target effect of morpholinos ${ }^{17}$. Notably, two different 
morpholinos targeting distinct regions of the bclwav gene were used (bclwavMO1 and bclwavMO2), whereas a control morpholino (5mis-MO, termed controlMO) was used as a negative control. The efficiency of the bclwavMOs was confirmed by immunoblotting of mitochondrial extracts of zebrafish embryos at 50\% epiboly (Fig. 3a). Bclwav knockdown induced major developmental abnormalities, and similar phenotypes were obtained with both morpholinos (Fig. 3b). Most bclwav morphants (86\%, $n=579$ ) (Fig. 3c and Supplementary Fig. S4a) were able to complete somitogenesis but showed
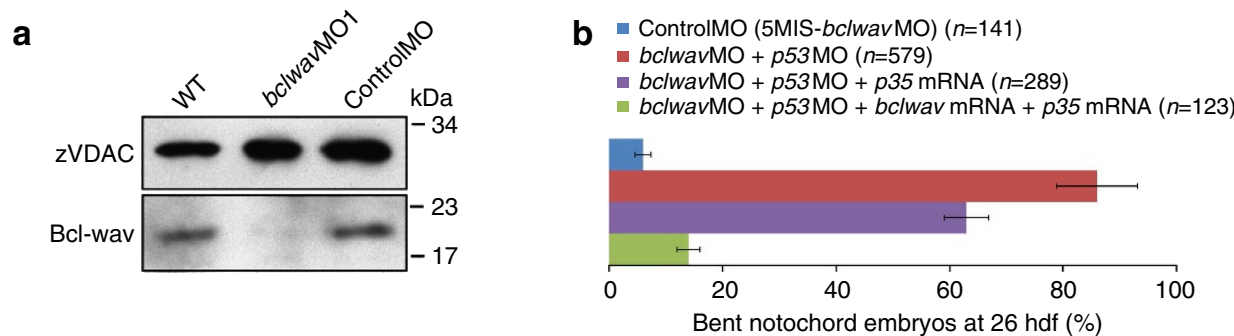
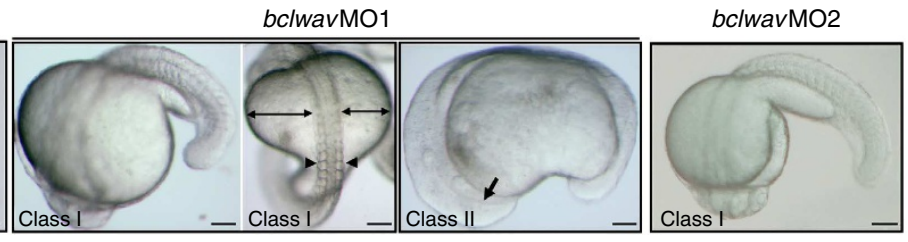

d

shh:gfp + bclwavMO

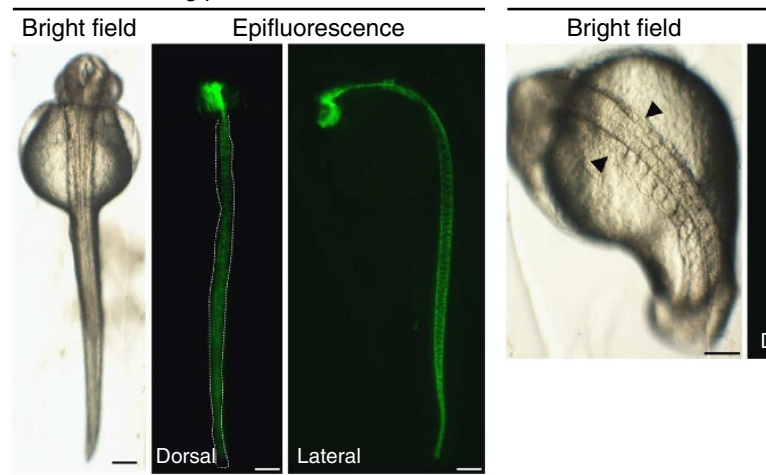

Epifluorescence

e
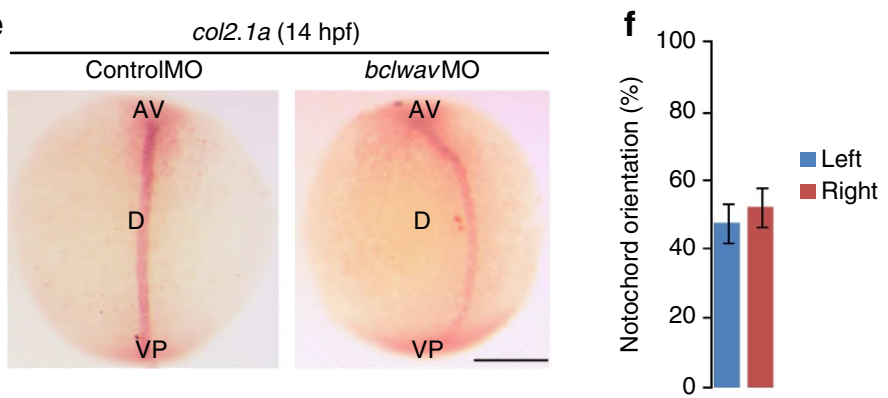

Figure 3 | Bclwav knockdown leads to major developmental defects. (a) Immunoblot analysis of bclwav morpholino efficiency in mitochondria purified from WT embryos and embryos injected with bc/wavMO1 and controlMO at 50\% epiboly ( $5 \mathrm{hpf}$ ) using anti-Bcl-wav polyclonal antibody. A specific signal that disappears following the injection of bclwavMO was detected. VDAC was used as an internal loading control. (b) Histograms representing the percentage of bent notochord phenotypes at $26 \mathrm{hpf}$ in controlMO (5Mis-MO + p53-MO) embryos versus bclwav morphants injected with bclwav or p35 mRNAs. The ectopic expression of $\mathrm{p} 35$ does not prevent the bclwavMO effect, indicating a caspase-independent phenomenon. Co-injection of bclwav mRNA (as well as p35 mRNA) together with bclwavMO restores normal development (mean \pm s.d.; three independent experiments). (c) Bclwav knockdown phenotype at $26 \mathrm{hpf}$. Embryos injected with negative controlMO were normal (left panel). In contrast, embryos injected with antisense morpholinos (bclwavMO1 + p53-MO or bclwavMO2 + p53-MO) show similar class I (77\%) and class II (9\%) abnormal phenotypes. Embryos with the class I phenotype (left and middle panels) exhibit abnormal somites (black arrowheads), as well as bent notochord and anteroposterior axis (two-ended arrows). The class II phenotype is characterized by a major developmental delay, showing the lack of anteroposterior differentiation at 26 hpf (right panel, black arrow). Scale bar, $200 \mu \mathrm{m}$. (d) Visualization of the bent notochord phenotype in the bclwav knockdown mutant using shh:gfp embryos compared with controlMO. In bclwav morphants, the notochord is shifted laterally (right panel), near the ear. Scale bar, $200 \mu \mathrm{m}$. (e) Col2.1a whole-mount in situ hybridization at $14 \mathrm{hpf}$. In contrast to controlMO embryos, bclwavMO embryos exhibit a deviation of the col2.1a signal relative to the anteroposterior axis. AP, animal pole; $\mathrm{D}$, dorsal; VP, vegetal pole. Scale bar, $200 \mu \mathrm{m}$. (f) Histogram representing the percentage of individuals with notochord deviation (to the right or to the left) in bclwavMO embryos at $26 \mathrm{hpf}$ (mean \pm s.d.; three independent experiments). Full scans of the western blots are provided in Supplementary Fig. S9. 
anteroposterior axis reduction and abnormal somites. Moreover, the use of SonicHedgehogGFP (shh:gfp) transgenic embryos showed that bclwav morphants exhibited misplaced and bent notochords, randomly shifted to either the right or the left, giving rise to a typical bending of the embryo at $24 \mathrm{hpf}$ (Fig. $3 \mathrm{~d}-\mathrm{f}$ ). These results were confirmed by in situ hybridization in $14 \mathrm{hpf}$ embryos using col2.1 as a probe, highlighting the curvature of the notochord (Fig. 3e). Contrary to bclwav morphants, most controls appeared to be normal (94\%, $n=141)$ (Fig. 3c). A minority of bclwav morphants (9\%) exhibited an even more severe phenotype, as illustrated by premature developmental arrest shortly after the beginning of somitogenesis. The specificity of the bclwav morphant phenotype was confirmed by co-injecting bclwav mRNA with bclwavMO. Under these conditions, the injected embryo phenotype ( $86 \%$ normal, $n=123$ ) was similar to that of the controls (Fig. 3c). In such complementation experiments, mRNA of the caspase inhibitor p35 was co-injected with bclwav mRNA to prevent apoptosis due to Bcl-wav protein accumulation. Interestingly, at later stages $(26 \mathrm{hpf})$, the somite alterations observed in bclwav morphants were correlated with a marked increase in caspase-dependent cell death events, as well as severe disorganization of the actin fibres in the somites, compared with control embryos (Supplementary Fig. S4b,c).

Overall, the results of these experiments suggest that bclwav knockdown causes developmental retardation and notochord bending, as well as somite malformation. Interestingly, ectopic expression of p35 failed to restore normal development (bent notochord embryos: 63\%, $n=289$ ) (Fig. 3c). These observations suggest that the bclwavMO phenotype did not result from caspase activation.

Bcl-wav controls $\mathrm{C} \& \mathrm{E}$ cell movements during gastrulation. In vertebrates, the co-ordinated movements of the blastomeres during $\mathrm{C} \& \mathrm{E}$ ensure the establishment of the anteroposterior axis and the embryo body plan. We hypothesized that the malformations observed at later stages in bclwav morphants might result from impaired gastrulation. We first analysed the anterior progression of the dorsal progenitor cells during gastrulation. Bclwav silencing resulted in a significant shortening of the embryos, suggesting a defect in morphogenetic movements during gastrulation (Fig. 4a,b). We next analysed the progression of mesoderm progenitors, which contributes to the formation of the notochord, by performing in situ hybridization at $75 \%$ epiboly and using no tail $(n t l)$ as a marker. In bclwav morphants, $n t l$-positive cells showed a disturbed anteroposterior distribution in the dorsal region (Fig. 4c). The length (l/L) and width $(\mathrm{w} / \mathrm{W})$ ratios of bclwav morphants $(\mathrm{l} / \mathrm{L}=0.45 \pm 0.04$ and $\mathrm{w} / \mathrm{W}=0.32 \pm 0.02)$ were significantly lower and higher, respectively, than those of the controls $(1 / L=0.61 \pm 0.03$ and $\mathrm{w} / \mathrm{W}=0.25 \pm 0.02$ ), suggesting a defect in the migration of these cells during gastrulation (Fig. 4d). Using time-lapse videomicroscopy, we specifically tracked the progression of the paraxial mesoderm, which undergoes $\mathrm{C} \& \mathrm{E}$ movements. The net progression of mesoderm cells towards the dorsal meridian axis was significantly affected in bclwav morphants (Fig. 4e). Analyses of their migration paths showed that, in contrast to the synchronously migrating control cells, the migration paths of many mesoderm cells in bclwav morphants were random (Fig. 4f). These results showed that although the mesoderm precursor cells migrated at a normal velocity in bclwav morphants, their progression towards the dorsal meridian axis was compromised. Because such alterations could be the consequence of cell autonomous or non-cell-autonomous phenomena, we set up a series of transplant experiments to observe the behaviour of morphant blastomeres in a wild-type (WT) environment. When implanted into WT receiving embryos, blastomeres from control donors were found to spread along the dorsal axis due to cellular intercalation (Fig. 4g). In contrast, transplanted blastomeres from morphant embryos were unable to undergo such spreading, which resulted in altered C\&E movements in the dorsal region of the morphant embryos (Fig. 4h). These results suggest that the origins of the observed defects were, at least in part, cell autonomous.

Bclwav knockdown alters F-actin dynamics during gastrulation. Cell migration depends strongly on actin microfilament dynamics. We thus monitored actin polymerization in control embryos and bclwav morphants using a green fluorescent protein (GFP)-tagged peptide that is able to bind F-actin (lifeactGFP). In control embryos, lateral mesodermal cells exhibited F-actin protrusions at the leading edge, which were oriented in the posterior-dorsal direction (Fig. 5a-d and Supplementary Movie 1). In contrast, F-actin protrusions in bclwav morphants were more randomly oriented (Fig. 5a-d and Supplementary Movie 2). Moreover, F-actin dynamics were also affected by bclwav silencing. In control embryos, F-actin protrusions were highly dynamic by polymerizing and depolymerizing periodically (average period: $80 \mathrm{~s}$ ), whereas these protrusions persisted for much longer periods in bclwav morphants, indicating that the dynamics of the protrusions were severely altered (Fig. 5a,b). Overall, these results suggest that bclwav silencing led to mesoderm C\&E migration defects as a consequence of impaired actin microfilament dynamics and polarity.

Bcl-wav sustains mitochondrial $\mathrm{Ca}^{2+}$ uptake via VDAC1. During gastrulation, Bcl-wav protein is exclusively mitochondrial. This led us to hypothesize that the observed C\&E defects could be due to mitochondrial dysfunction. Surprisingly, none of the mitochondrial parameters analysed, including mitochondrial transmembrane potential $(\Delta \Psi \mathrm{m})$, ROS production, mitochondrial respiration or cytochrome- $c$ release, appeared to be affected in bclwav morphants (Supplementary Fig. S5). However, when mitochondrial $\mathrm{Ca}^{2}+$ levels were measured using a dedicated fluorescent dye (Rhod-FF), we detected a significant decrease in mitochondrial $\mathrm{Ca}^{2+}$ content compared with control embryos (Fig. 6a-c). Moreover, this mitochondrial $\mathrm{Ca}^{2}+$ decrease was correlated with an increase in cytosolic $\left[\mathrm{Ca}^{2+}\right]$ and a decrease in the mitochondria to cytosolic calcium ratio, suggesting that Bclwav might control $\mathrm{Ca}^{2+}$ fluxes between mitochondria and the cytosol (Fig. 6a-c and Supplementary Movies 3 and 4). To test this hypothesis further, we evaluated the effect of ectopic Bcl-wav expression on mitochondrial $\mathrm{Ca}^{2}+$ uptake following an artificial increase in cytosolic $\mathrm{Ca}^{2+}$ levels in HeLa cells. Bcl-wav expression significantly increased mitochondrial $\mathrm{Ca}^{2+}$ uptake (Fig. 6d,e). We next evaluated the effect of bclwav knockdown on the capacity of purified mitochondria from zebrafish embryos to take up exogenous $\mathrm{Ca}^{2}+(20 \mu \mathrm{M})$. Bclwav-deficient mitochondria had a decreased $\mathrm{Ca}^{2+}$ uptake capacity compared with control mitochondria (Fig. 6f,g). VDAC1 is a major component of the apoptosis machinery; the pharmacological inhibition of VDAC using $2 \mathrm{mM}$ NADH completely abolished $\mathrm{Ca}^{2+}$ entry into mitochondria, illustrating the critical role of VDAC as a $\mathrm{Ca}^{2+}$ channel $5,18,19$ (Fig. 6f,g). It was previously shown that $\mathrm{Bcl}-2$ proteins, including Bcl-xL, could interact with $\mathrm{VDAC}^{20}$ and modulate its activity via the $\mathrm{BH} 4$ domain $^{21}$. We thus evaluated whether Bcl-wav can interact with VDAC1. To this end, Flag-tagged Bcl-wav was ectopically expressed in zebrafish embryos. Immunoprecipitation assays performed using purified mitochondria from embryos overexpressing Flag-Bcl-wav showed that full-length $\mathrm{Bcl}-\mathrm{wav}$ interacted with endogenous zVDAC1 (Fig. 6h). Interestingly, Bcl-wav was also found to interact with 
a

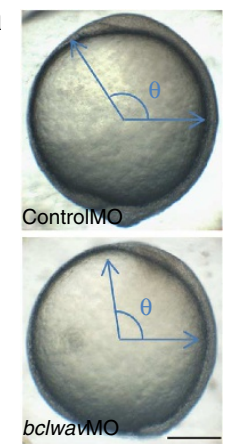

b

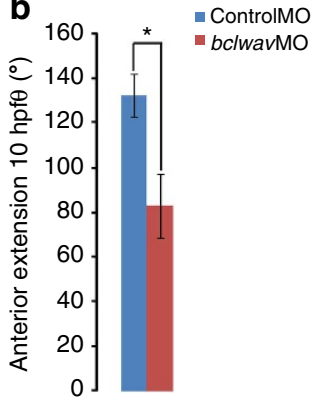

e

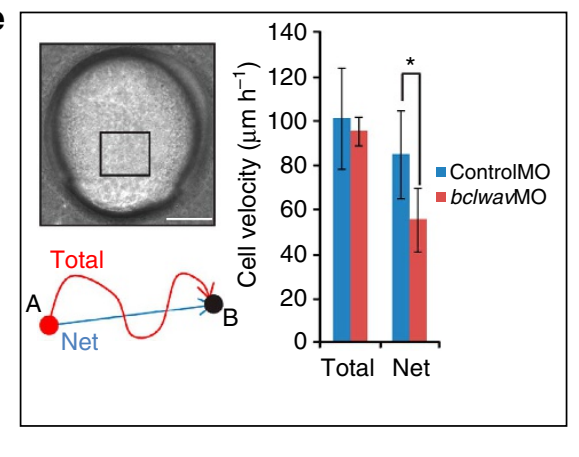

g

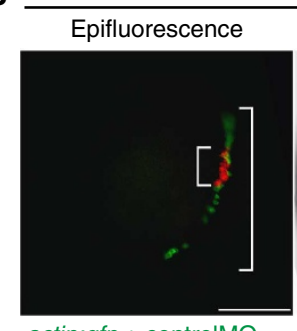

actin:gfp + controlMO

bclwavMO + rhodamine

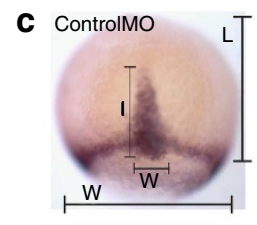

bclwavMO
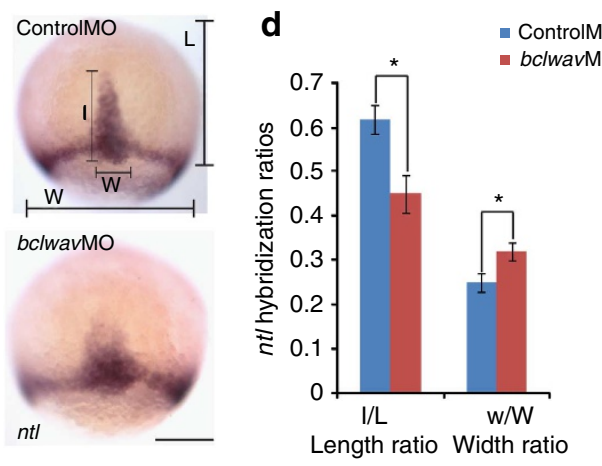

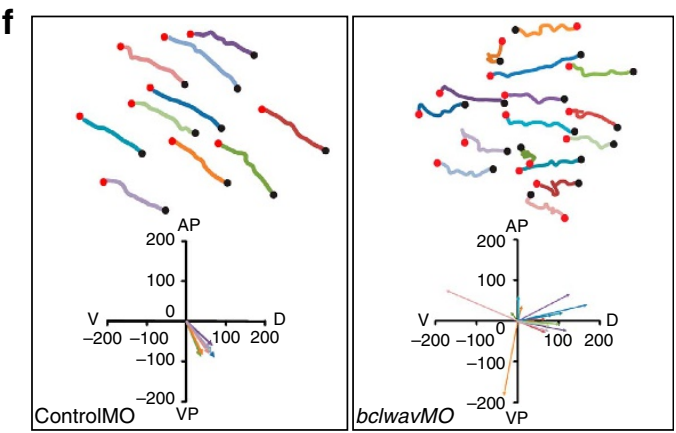

h

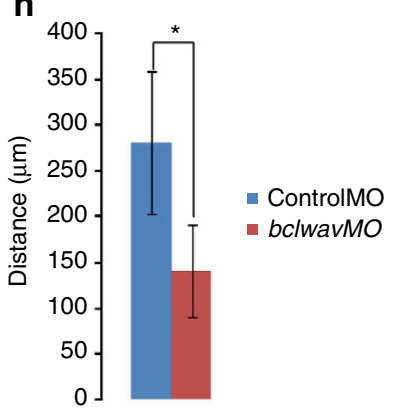

Figure 4 | Effect of bclwav knockdown on cell movement during C\&E movements. (a) Representative bright field images of controlMO and bclwavMO at the tail bud stage, showing the delay in the progression of dorsal progenitors as measured by the angle between the head and the equator $(\theta)$. Scale bar, $200 \mu \mathrm{m}$. (b) Histograms quantifying the $\theta$ angle difference between control embryos and bclwav morphants. Bclwav silencing induces a significant reduction in anterior extension (mean \pm s.d.; three independent experiments; ${ }^{\star} P<0.01$, Student's $t$-test). (c) $N t /$ whole-mount in situ hybridization at $75 \%$ epiboly. The $n t$-positive mesoderm cells in bclwav morphants show disturbed anteroposterior migration at the dorsal pole relative to controlMO embryos. $\mathrm{Nt}$-positive signal length (I/L) and width (w/W) ratios are used to calculate mesoderm progression. Scale bar, $200 \mu \mathrm{m}$. (d) Histograms representing the $\mathrm{I} / \mathrm{L}$ and $\mathrm{w} / \mathrm{W}$ ratios of controlMO embryos and bclwav morphants at the $75 \%$ epiboly stage (mean $\pm \mathrm{s}$.d.; three independent experiments; ${ }^{\star} P<0.01$, Student's $t$-test). (e) Histograms representing the quantification of the total velocity (total distance, $\mu \mathrm{m} \mathrm{h}^{-1}$ ) and net velocity (net dorsal progression, $\mu \mathrm{m} \mathrm{h}^{-1}$ ) of the lateral mesodermal cells during C\&E movements (at 75\% epiboly). The net velocity of the mesodermal cells is significantly lower in bclwavMO than in control embryos (mean \pm s.d.; three independent experiments; ${ }^{\star} P<0.01$, Student's $t$-test). Scale bar, $200 \mu \mathrm{m}$. (f) Cell paths and vector projections of isolated mesodermal cells observed during C\&E movements at the $75 \%$ epiboly stage in embryos injected with controlMO or bclwavMO. Compared with the control cells, which migrate synchronously, bclwavMO lateral mesodermal cells exhibit more random migration paths. Images and diagrams are oriented as follows: AP, animal pole; D, dorsal; V, ventral; and VP, vegetal pole. (g) Observation of GFP-expressing cells injected with controlMO and morphant (bclwavMO + rhodamine) cells transplanted into WT embryos. Control cells (green) show a greater distribution along the dorsal axis than the bclwav-silenced cells (red). Scale bar, $200 \mu \mathrm{m}$. (h) Histograms showing the distance covered by controlMO cells versus bclwav-silenced cells (controlMO: $n=13$ embryos and bclwavMO: $n=10$ embryos) (mean \pm s.d.; ${ }^{\star} P<0.01$, Student's $t$-test).

endogenous VDAC1 in HeLa cells (Fig. 6i), which might be expected because zebrafish and human VDAC1 are highly conserved (amino acid sequence identity: 85.5\%). To determine whether Bcl-wav interacted with VDAC1 via its BH4 domain, immunoprecipitation assays in HeLa cells were also performed with Bcl-wav-deletion mutants in addition to full-length Bcl-wav. As shown in Fig. 6i, full-length Bcl-wav and Bcl-wav $\Delta \mathrm{TM}$ interacted with VDAC1. Although no interaction with $\triangle \mathrm{BH} 4 \mathrm{Bcl}-$ wav was detected, the BH4 domain alone interacted with VDAC1.
In addition, in bclwav morphants, the $\Delta \mathrm{BH} 4 \mathrm{Bcl}-$ wav-deletion mutant neither restored normal development (Fig. 6j) nor prevented increases in cytosolic $\mathrm{Ca}^{2+}$ (Fig. 6k), in contrast to fulllength Bcl-wav. This result suggests that the Bcl-wav/zVDAC1 interaction was functionally significant. Together, these findings suggest that $\mathrm{Bcl}-$ wav may control mitochondrial $\mathrm{Ca}^{2+}$ uptake via zVDAC1 during zebrafish gastrulation. Most interestingly, zBcl-xL completely reversed the effect of Bcl-wav on mitochondrial $\mathrm{Ca}^{2}+$ uptake (Fig. $6 c$,d). Indeed, the ability of $\mathrm{zBcl}-\mathrm{xL}$ to 

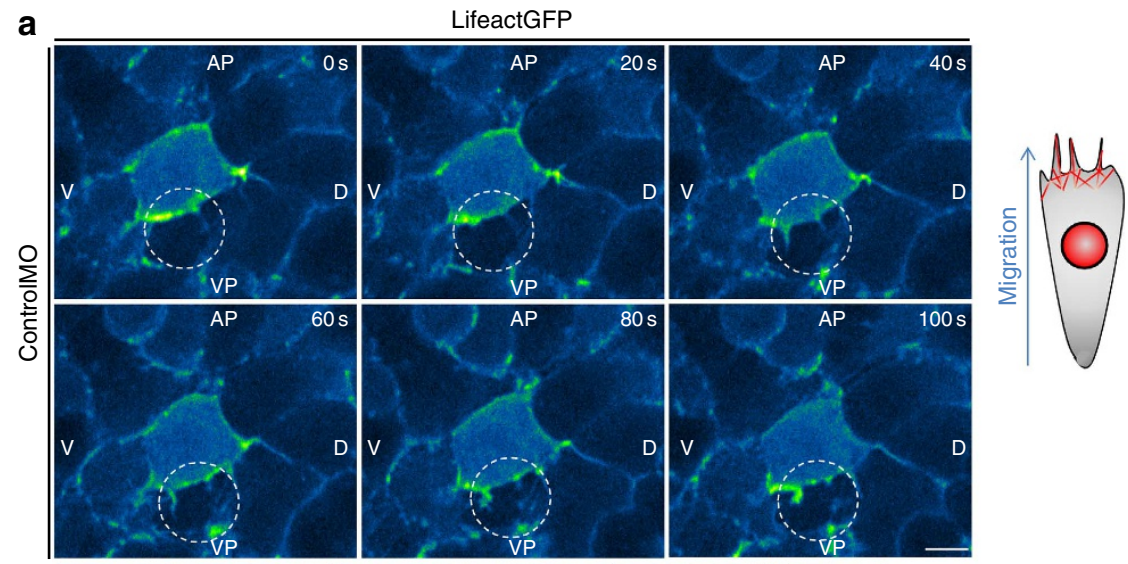
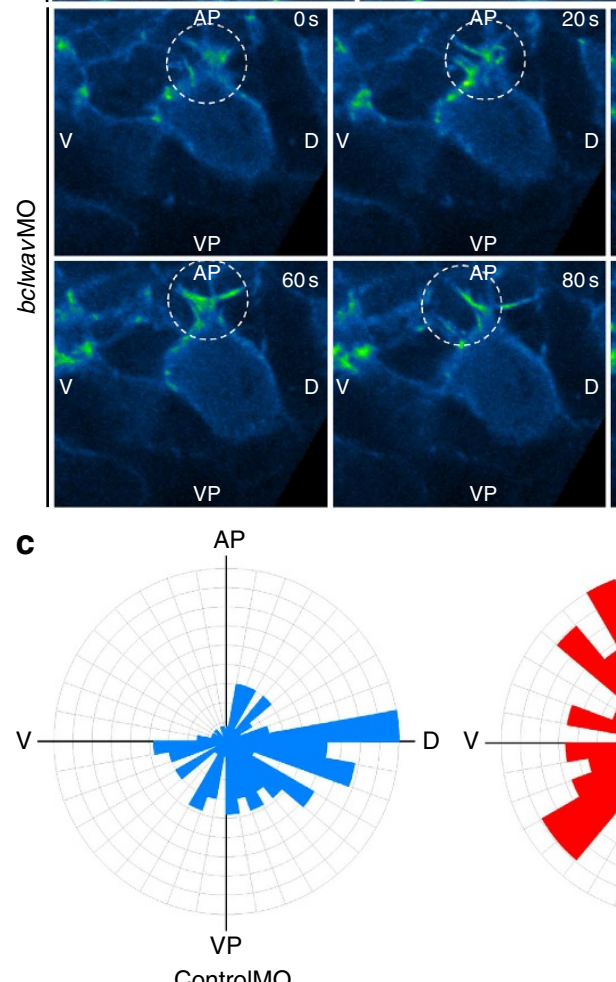

ControlMO

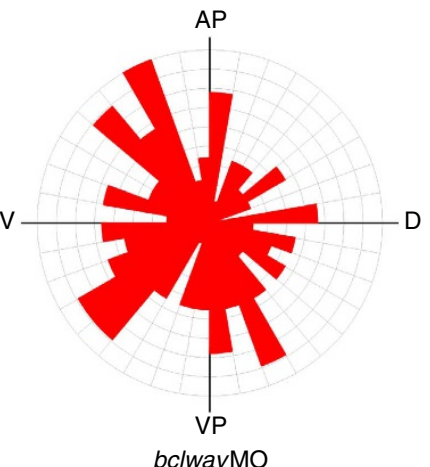

bclwavMO

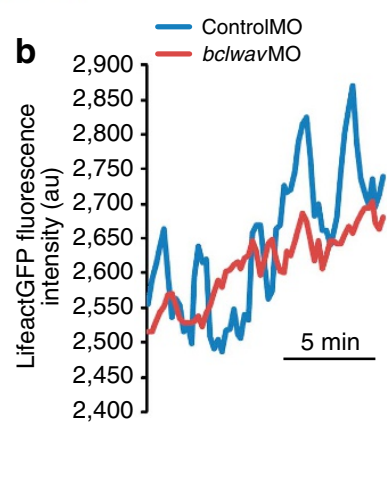

d

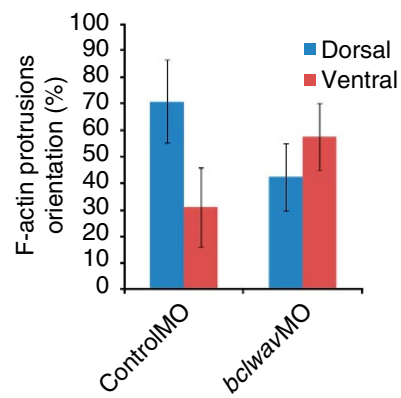

Figure 5 | Bclwav knockdown disrupts actin protrusion dynamics and orientation. (a) Confocal spinning disk microscopy analysis of F-actin dynamics in controlMO and bclwav morphants expressing lifeactgfp at the 75\% epiboly stage. F-actin-enriched cell protrusions are essential for cell migration. Cells from bclwavMO-injected embryos show altered F-actin polymerization positioning and dynamics (white circles). Image acquisition was performed on a single confocal section to visualize polymerized actin in lateral mesodermal cells. Scale bar, $10 \mu \mathrm{m}$. (b) Graph representing the fluorescence intensity of LifeactGFP-positive cells over 20 min of tracking. Control cells show periodic increases and decreases in fluorescence intensity corresponding to F-actin polymerization and depolymerization. In bclwav-silenced cells, these F-actin dynamics are strongly altered. (c) Circular rose diagrams of controlMO versus bclwavMO embryos representing the orientation of actin protrusions. These diagrams show the frequency of protrusions in a given orientation. The directionality of the F-actin protrusions is more random in bclwavMO embryos than in controlMO embryos. Diagrams are oriented as follows: AP, animal pole; D, dorsal; V, ventral; VP, vegetal pole. (d) Histograms representing F-actin protrusion orientations. The F-actin protrusions of control embryos are mainly oriented in the dorsal quadrant, whereas those in bclwav morphants are inverted (that is, in the ventral quadrant) (mean $\pm s . d . ; n=111$ and 141 cells for controlMO and bclwavMO, respectively; three independent experiments).

counteract the effect of Bcl-wav on $\mathrm{Ca}^{2+}$ uptake might also depend on VDACl, given that Bcl-xL has previously been shown to bind to $\operatorname{VDAC}^{21-23}$.

C\&E movements strongly depend on mitochondrial $\mathrm{Ca}^{2+}$ uptake. $\mathrm{Ca}^{2+}$ acts as a major intracellular messenger that controls different cellular processes and has a critical role during zebrafish development ${ }^{12}$. It has been clearly established that cytosolic $\mathrm{Ca}^{2+}$ levels control actin dynamics and contribute to C\&E movements in Xenopus and zebrafish ${ }^{24,25}$. Our results strongly suggest that the migration defects observed following bclwav silencing were due to a decrease in mitochondrial $\mathrm{Ca}^{2}+$ uptake and subsequent $\mathrm{Ca}^{2+}$ accumulation in the cytosol.

To confirm this hypothesis, we directly tested whether disrupting mitochondrial $\mathrm{Ca}^{2+}$ uptake would lead to the same C\&E alterations as observed in bclwavMO. To this end, we knocked down the $m c u$, which was recently reported to be the 
major $\mathrm{Ca}^{2+}$ carrier in the inner mitochondrial membrane ${ }^{6,7}$. Mcu silencing increased cytosolic $\mathrm{Ca}^{2}+$ levels and decreased mitochondrial $\mathrm{Ca}^{2+}$ levels (Fig. 7a), which correlated with major developmental alterations, including notochord deviation and anteroposterior axis reduction (Fig. $7 \mathrm{~b}, \mathrm{c}$ ). Moreover, mcu knockdown also compromised blastomere migration (Fig. 7d) and altered F-actin dynamics and orientation (Fig. 7f,g), thus phenocopying the bclwav knockdown (Supplementary Movie 5). It should be noted that the concurrent silencing of $m c u$ and bclwav did not cause additive developmental defects (Supplementary Fig. S6).

Conversely, we observed that the 'bent notochord' phenotype of the bclwav and mcu morphants could be rescued by coinjecting the EGTA derivative Bapta-1 AM, an efficient chelator of free $\mathrm{Ca}^{2+}$ (Fig. 7e). These results suggest that the developmental abnormalities observed in morphant embryos were the direct consequence of $\mathrm{Ca}^{2+}$-trafficking alterations in blastomeres. During early zebrafish development, $\mathrm{Ca}^{2+}$ signalling is primordial during morphogenetic movements ${ }^{26}$. Moreover, C\&E movements appear to be partially under the control of the Wnt/ PCP pathway and small GTPases such as RhoaA; thus, these movements could be regulated by calcium signalling ${ }^{9,27}$. We therefore investigated the effect of $m c u$ knockdown on RhoA activation in human cells using a Rhotekin pull-down experiment. The efficiency of the small interfering RNA used in these experiments was previously validated in cellulo (Supplementary Fig. S7). The level of active RhoA was significantly lower in $\mathrm{mcu}$-silenced cells than in control cells (Supplementary Fig. S8). Together, these results suggest that the cytosolic calcium accumulation observed in bclwav and $m c u$ morphants may be responsible, at least in part, for the observed alteration of F-actin remodelling.

Bcl-wav/VDAC1/MCU complex controls mitochondrial $\mathrm{Ca}^{2+}$ uptake. Finally, we performed a set of in cellulo experiments to better characterize the effect of Bcl-wav on mitochondrial $\mathrm{Ca}^{2+}$ uptake. Bcl-wav was unable to increase mitochondrial $\mathrm{Ca}^{2+}$ uptake (Fig. 8a,b) in an $m c u$ - or/and vdac1-null background, suggesting that VDAC1 and MCU may form a multiprotein $\mathrm{Ca}^{2+}$ channel that controls $\mathrm{Ca}^{2+}$ trafficking and blastomere movements during gastrulation. Indeed, Bcl-wav directly interacted with both VDAC1 and MCU, as shown by co-immunoprecipitation experiments in HeLa cells, suggesting that such interactions may also occur in the developing zebrafish (Fig. 8c,d). Importantly, contrary to the effects on mitochondrial $\mathrm{Ca}^{2+}$ uptake, the pro-apoptotic effect of Bcl-wav seems to be MCU independent. Indeed, Bcl-wav can still activate caspase 3 and induce PARP cleavage in $m c u$ knockdown cells (Fig. 8e) or in $m c u$ morphant embryos (Fig. 8f), and $\Delta$ BH4Bcl-wav, which does not interact with VDAC1, was fully pro-apoptotic (Supplementary Fig. S2c). Consistent with these observations, a Bapta-1 AM probe was unable to rescue the embryonic mortality induced by bclwav mRNA (Fig. 8g).

Taken together, these results show that Bcl-wav enhances $\mathrm{Ca}^{2+}$ uptake into the mitochondria via VDAC1 and MCU and may accelerate apoptosis by activating Bax. During gastrulation, Bcl-wav and MCU serve as key factors in C\&E movements by controlling mitochondrial $\mathrm{Ca}^{2+}$ trafficking and the dynamics of F-actin protrusions in migrating blastomeres (Fig. 8h).

\section{Discussion}

Here we report the identification and functional characterization of Bcl-wav, a new member of the multidomain Bcl-2 family of apoptosis regulators. This gene was identified using profile-based hidden Markov models, combining sequence and three- dimensional structure information ${ }^{15}$. Interestingly, Bcl-wav was found in aquatic egg-laying species (teleosts and anurans) but not in mammals or birds, suggesting that Bcl-wav was evolutionarily selected for a specific role in the particular reproductive process of these egg-laying species. Indeed, some $\mathrm{Bcl}-2$ members seem to have specialized roles in the control of oocyte survival and early embryo development ${ }^{13,28-30}$. Combined bioinformatic and functional analyses identified Bcl-wav as a new multidomain Bcl-2 member ${ }^{31}$. We showed that Bcl-wav is able to bind to Bax and subsequently induce caspase activation. Thus, Bcl-wav seems to be an atypical member of the subfamily of multidomain death accelerators. The Bcl-wav protein is mostly a mitochondrial resident, suggesting that it may share some functional similarities with Bak, a multidomain protein that is not expressed during zebrafish development ${ }^{11}$. In this regard, Bcl-wav is able to interact with VDAC1, a property shared with Bak ${ }^{9,32}$. VDAC1 is known to permit $\mathrm{Ca}^{2+}$ transfer from the cytosol to the mitochondrial matrix ${ }^{19}$. This suggests that $\mathrm{Bcl}-\mathrm{wav}$ could have a role in the control of intracellular $\mathrm{Ca}^{2+}$ fluxes. Bclwav knockdown resulted in a marked decrease in mitochondrial $\mathrm{Ca}^{2+}$ levels, together with an increase in cytosolic $\mathrm{Ca}^{2+}$. Interestingly, knockdown of $\mathrm{mcu}$ also induced marked increases in cytosolic $\mathrm{Ca}^{2+}$ and phenocopied the bclwav knockdown phenotype, which not only demonstrates the critical role of MCU in vertebrate development but also suggests the existence of a multiprotein $\mathrm{Ca}^{2+}$ channel composed of VDAC1 and MCU. Indeed, Bcl-wav can interact with both VDAC1 and MCU, and these proteins can also interact with each other. In addition, the effect of Bcl-wav on mitochondrial calcium uptake depends on both VDAC1 and MCU. Thus, at the molecular level, it may be speculated that $\mathrm{Bcl}-$ wav maintains the VDAC1/MCU channel in an 'open state', allowing $\mathrm{Ca}^{2+}$ entry into the mitochondrial matrix. In this way, the fine-tuning of mitochondrial $\mathrm{Ca}^{2+}$ fluxes might be ensured by the combined effects of apoptosis accelerators, including Bclwav, and apoptosis inhibitors, such as Bcl-xL, on the permeability of the VDAC1 $\mathrm{Ca}^{2+}$ channel. In fact, Bax itself was reported to interact with $\mathrm{VDAC}^{33}$, but also $\mathrm{Bcl}-\mathrm{xL}^{21}$ that can modulate its permeability ${ }^{22}$. Our data support the model that $\mathrm{Bcl}-\mathrm{xL}$ is able to inhibit massive $\mathrm{Ca}^{2+}$ entry into mitochondria. In this respect, it would be interesting to evaluate the existence of a 'ménage à trois' between Bcl-wav, Bcl-xL and VDAC1.

The observed phenotypes of bclwav and mcu morphants may tentatively be attributed, at least in part, to the observed effects on cytosolic and mitochondrial $\mathrm{Ca}^{2+}$ pools. Indeed, during zebrafish gastrulation, C\&E movements appear to be partially under the control of the Wnt/PCP pathway ${ }^{34,35}$. This pathway activates small GTPases such as RhoA and Rac1, which are responsible for F-actin dynamics and polarization ${ }^{36}$. Given that RhoA activation is decreased in $m c u$ null cells, we hypothesize that bclwav and $m c u$ silencing in zebrafish impair F-actin dynamics by altering Rho GTPase activity. Indeed, it has been demonstrated that an increase in cytosolic $\mathrm{Ca}^{2+}$ modulates Racl and RhoA activities, leading to alterations in cell migration and reversing the orientation of protrusions in neuronal cells ${ }^{36}$. Interestingly, components of the Wnt/PCP pathway can also modulate $\mathrm{Ca}^{2+}$ signalling 37,38 . These data are consistent with observations in zebrafish, showing that throughout gastrulation $\mathrm{Ca}^{2+}$ waves occur in the vicinity of the dorsal axis of the embryo, where C\&E movements take place ${ }^{39}$. During these $\mathrm{Ca}^{2+}$ waves, cells are exposed to high $\mathrm{Ca}^{2+}$ concentrations, which may be detrimental; thus, mitochondrial $\mathrm{Ca}^{2+}$ uptake is presumably critical for maintaining $\mathrm{Ca}^{2+}$ homoeostasis in migrating blastomeres. In bclwav and mcu morphants, mitochondrial $\mathrm{Ca}^{2}+$ uptake appears to be compromised, as suggested by decreased mitochondrial $\mathrm{Ca}^{2+}$ levels; this may in turn explain the observed cytosolic $\mathrm{Ca}^{2+}$ increase. Thus, bclwav knockdown may alter C\&E 
movements via its effects on temporal and spatial $\mathrm{Ca}^{2+}$ signalling in the gastrula. This hypothesis accounts for the observed disruption of the distribution of $n t l$-positive cells in these morphants and the subsequent malformations. It was recently shown that embryos that are deficient in components of the Wnt/PCP pathway and thus have abnormal C\&E movements also exhibited defects in somite and notochord formation ${ }^{40}$.

Together, our results support the hypothesis that Bcl-wav orchestrates morphogenetic movements by acting on intracellular $\mathrm{Ca}^{2+}$ trafficking via its interactions with VDAC1/MCU. These data raise the possibility that the primary role of $\mathrm{Bcl}-2$ proteins during development might be to maintain cytoskeletal dynamics by regulating intracellular $\mathrm{Ca}^{2+}$ fluxes. From an evolutionary point of view, the Bcl-2 family might be linked to the appearance of gastrulation in metazoans.

\section{Methods}

Zebrafish care. Experimental procedures followed the recommendations of the French Council on Animal Care (Arrêté du 27 décembre 1994). Protocols were approved by the Committee for animal experimentation Université Claude Bernard Lyon 1.

Zebrafish $\left(\mathrm{AB} / \mathrm{TU}\right.$ and $\mathrm{AB} / \mathrm{TL}$ strains) were raised and maintained at $28.5^{\circ} \mathrm{C}$ according to the standard procedures. Embryos were collected after fertilization and injected at the one-cell stage. a

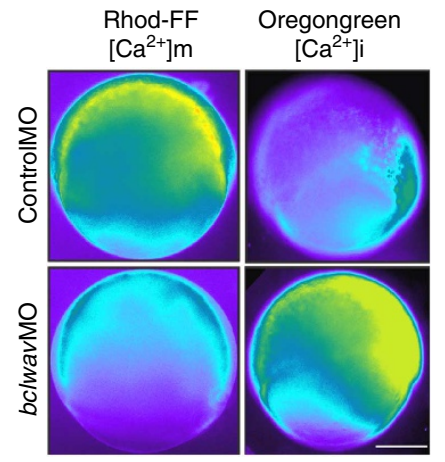

d

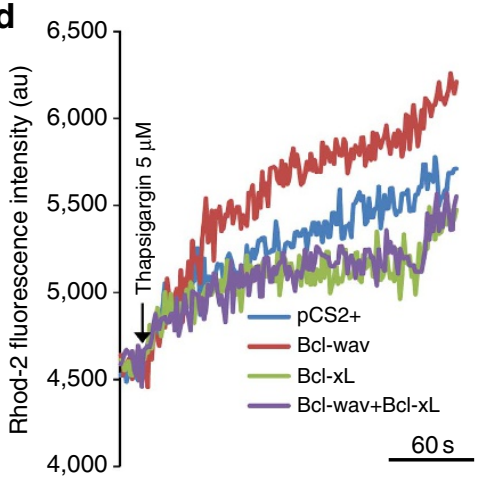

g

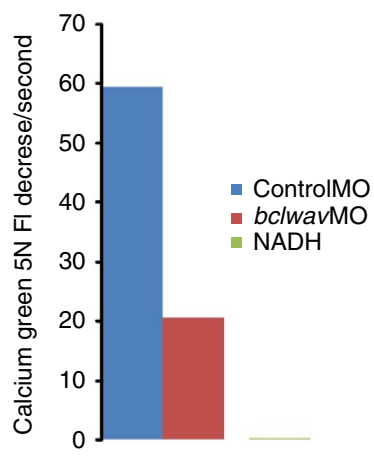

j b

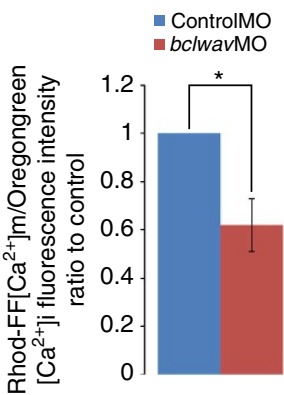

C

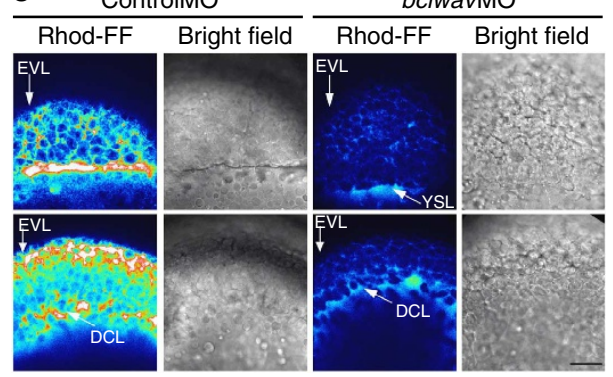

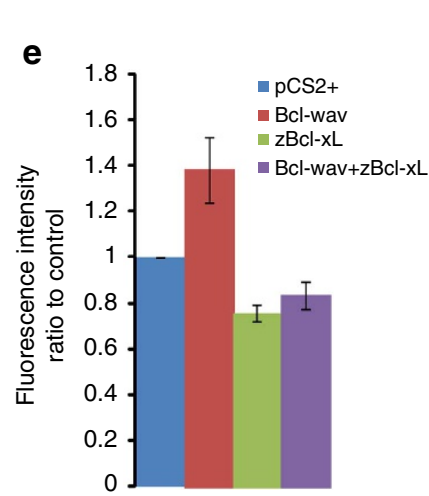

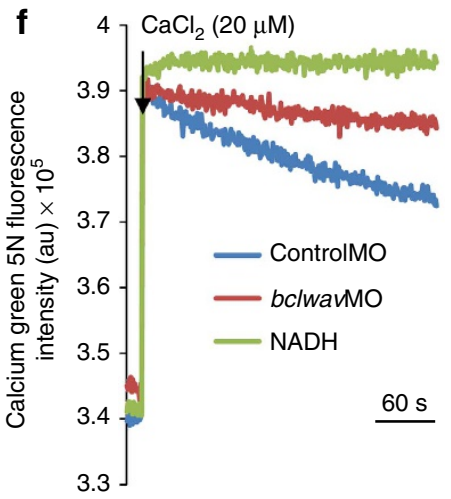

h

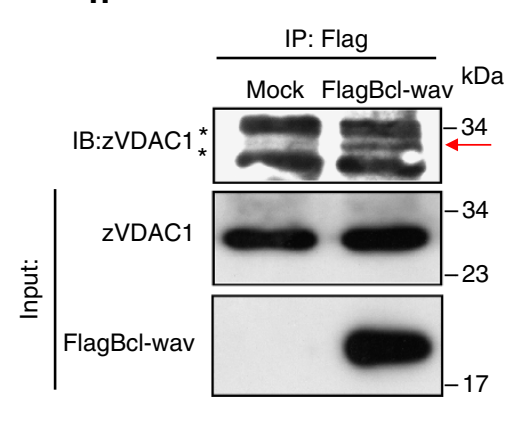

i

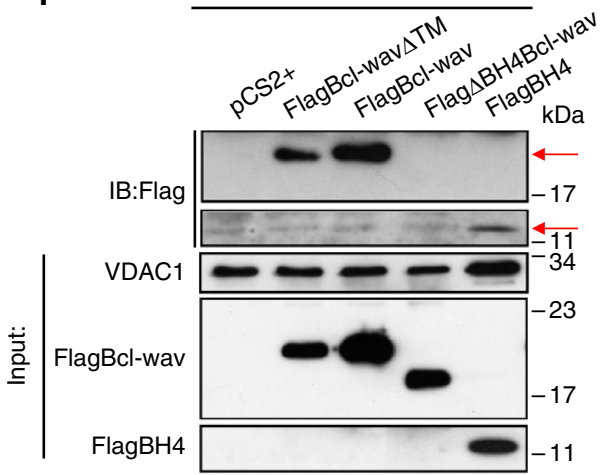

$\mathbf{k}$

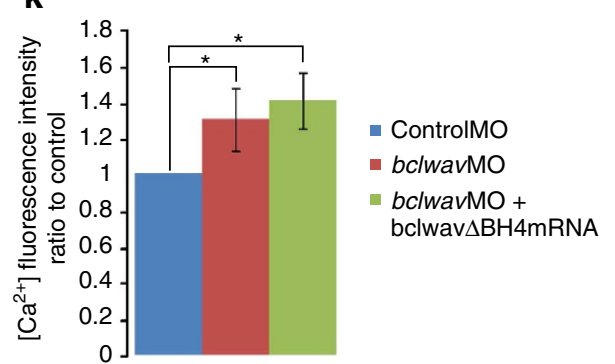


Morpholinos and mRNA. Morpholinos were designed according to the manufacturer's recommendations (Gene Tools, Philomath, OR, USA): bclwavMO1 (5'-CATCAGCTCAGATACACACAGGTCA-3') hybridizes with bclwav mRNA from position -22 to +3 relative to the start codon; bclwavMO2 (5'-TCACAG CGTCGTCTGACCGTCCCAT-3') hybridizes with bclwav mRNA from position +1 to position +25 . We used the bclwavMO1 with five mismatches (underlined) as negative control: 5 mis-MO (5' ${ }^{\prime}$-CGTCAGATCGGATACTCACAGGTTA- $\left.3^{\prime}\right)$.

Other morpholinos included $p 5 \overline{3-\mathrm{MO}}\left(5^{-}\right.$-GC $\overline{\mathrm{G}} \mathrm{CCATT} \overline{\mathrm{G}} \mathrm{CTTTGCA \overline {A } G A A}$ TTG-3'), mcuMO (5'-CATCTCTGAAGTGAAACCCGGCCGA-3') and $m c u$-5mis-MO (5'-GAGCTATGAATTGAAACTCGGACGA- $\left.{ }^{\prime}\right)$. Details regarding bclwav cloning (primer sequences are listed in Supplementary Table S1) and small interfering RNA experiments are provided in Supplementary Methods.

Bcl-wav protein and antibody production. The Bcl-wav protein (residues 1-177) was produced in BL-21 (DE3) bacteria, purified on a Ni-NTA-agarose column ${ }^{41}$ and subsequently used for polyclonal antibody production (Valbex, Villeurbanne). Rabbit serum immunoglobulin was purified on a protein A-agarose affinity column $^{42}$

In situ hybridization. For bclwav expression analysis, three probes were used: two were partial and non-overlapping (bclwav ${ }^{1-293}$ from position +1 to +293 and $b c l w a v^{300-600}$ from position +300 to +600 ), and one represented full-length bclwav. Briefly, digoxigenin-labelled riboprobes were synthesized from pCS2 + bclwav, pCS2 + bllwav $^{1-293}, \mathrm{pCS} 2+$ bclwav $^{300-600}, \mathrm{pCS} 2+n t l$ and $\mathrm{pCS} 2+$ col2.1a. After permeabilization, embryos were hybridized overnight. Then, embryos were incubated with an anti-digoxigenin antibody (Roche; 1:10,000), stained and observed under a Leica MZ6 stereo-microscope ${ }^{43}$.

Subcellular fractionation. All steps were carried out at $4^{\circ} \mathrm{C}$. Blastomere mitochondria, mitochondria and ER localized to the YSL of different embryo stages (before mid-blastula transition, sphere, $30 \%, 50 \%$ and $75 \%$ epiboly progression) were purified as follows: 100 embryos were added to $1 \mathrm{ml}$ of cold MB buffer (210 mM mannitol, $70 \mathrm{mM}$ sucrose, $1 \mathrm{mM}$ EDTA and $10 \mathrm{mM}$ HEPES ( $\mathrm{pH} 7.5$ ) containing protease inhibitors), and the yolk sac was disrupted by pipetting up and down. Embryos were centrifuged for $2 \mathrm{~min}$ at $300 \mathrm{~g}$ to separate blastomeres and yolk cells. The pellet, containing blastomeres, was resuspended in $1 \mathrm{ml}$ of $\mathrm{MB}$ buffer, and the cells were disrupted by shearing with a 1-ml syringe and a $26 \mathrm{G} \times 2 / 3$ needle 50 times. The disrupted blastomeres and yolk cells were then centrifuged twice at $1,500 \mathrm{~g}$ for $5 \mathrm{~min}$ to eliminate nuclei and finally centrifuged at $10,600 \mathrm{~g}$ to pellet the mitochondria. Mitochondria were washed with $1 \mathrm{ml}$ of MB buffer and resuspended in an appropriate volume of MB buffer for further analyses. The supernatant was centrifuged at $100,000 \times g$ for $1 \mathrm{~h}$, and the pellet containing the ER fraction was resuspended in RIPA buffer for further analysis.

Cell death assays. To study Bcl-wav pro-apoptotic activity, HeLa cells were transiently transfected with appropriate constructs and treated with dimethyl sulphoxide (DMSO) or $10 \mu \mathrm{M}$ thapsigargin $24 \mathrm{~h}$ later. Then, $24 \mathrm{~h}$ after drug treatment, cell death was quantified by annexin-V-Cy3 (Biovision) staining according to the manufacturer's protocols followed by flow-cytometry analysis using a FACScan (Becton Dickinson). Data were processed using CellQuest Pro software.

To analyse the effect of Bcl-wav on caspase activation, HeLa cells were transiently transfected with appropriate constructs alone or in combination. Twenty-four hours later, caspase-positive cells were labelled using the FLICA detection kit (ImmunoChemistry Technologies LLC). Pycnotic nuclei were labelled using Hoechst 33342. Cells were observed under a fluorescent microscope, and the percentages of caspase-positive and Hoechst-positive cells were determined $(\sim 100$ cells per field).

For terminal deoxynucleotidyl transferase-mediated dUTP nick end-labelling assays, embryos were fixed in $4 \%$ paraformaldehyde in PBS overnight at $4{ }^{\circ} \mathrm{C}$, washed at room temperature and stored in methanol at $-20^{\circ} \mathrm{C}$. After progressive rehydratation in PBS, embryos were washed four times in PBS, $0.1 \%$ Tween 20 (5 min per wash). Permeabilization was performed for $15 \mathrm{~min}$ in PBS, $0.1 \%$ Triton $\mathrm{X}-100$ and $0.1 \%$ sodium citrate at $+4^{\circ} \mathrm{C}$, cell death was quantitated using the POD in situ cell death detection kit (Roche). Cell death was detected by measuring the incorporation of FITC-labelled nucleotides by confocal microscopy.

For zebrafish caspase 3 assay, embryos (50\% epiboly) were fixed in $4 \%$ paraformaldehyde overnight at $4{ }^{\circ} \mathrm{C}$ and stored in $100 \%$ methanol at $-20^{\circ} \mathrm{C}$. After progressive rehydratation in PBS, embryos were washed three times for $10 \mathrm{~min}$ in PBS $+0.1 \%$ Tween (PBST) and subsequently soaked for $1 \mathrm{~h}$ in blocking solution (10\% heat-inactivated FBS and 3\% BSA in PBST). Embryos were then incubated overnight at $+4{ }^{\circ} \mathrm{C}$ with anti-activated caspase 3 antibody $(1 / 500)$. Following three washes in PBST, $0.3 \%$ Triton and $1 \%$ DMSO, embryos were soaked in blocking solution for $1 \mathrm{~h}$. Embryos were then incubated overnight at $+4{ }^{\circ} \mathrm{C}$ with a goat anti-rabbit red fluorescent antibody (1/200 in blocking solution). Embryos were washed three times in $0.3 \%$ Triton X-100 and $1 \%$ DMSO in PBST, incubated with Hoechst $33342(1 / 10,000)$ for 30 min, washed with $1 \times$ PBS and stored in $80 \%$ glycerol. Embryos were analysed using a Nikon TE300 fluorescence microscope.

Immunoprecipitation. For zebrafish embryo immunoprecipitation experiments, mitochondria from embryos injected with or without Flag-Bcl-wav mRNA at 30\% epiboly were purified as described above. Mitochondria were resuspended in 1\% CHAPS buffer (1\% CHAPS, $150 \mathrm{mM} \mathrm{NaCl}, 50 \mathrm{mM}$ Tris ( $\mathrm{pH}$ 7.4) and protease inhibitors). Extracts were precleared with protein G-Sepharose beads for $2 \mathrm{~h}$ at $4^{\circ} \mathrm{C}$, and then $100 \mu \mathrm{g}$ of mitochondria was incubated with $5 \mu \mathrm{g}$ of anti-Flag M2 overnight. Extracts were then incubated for $3 \mathrm{~h}$ with $15 \mu \mathrm{l}$ of protein G-Sepharose beads and centrifuged for $5 \mathrm{~min}$ at 5,000 r.p.m. Collected beads containing immunoprecipitated fractions were washed three times in CHAPS buffer, resuspended in SDS-PAGE sample buffer and analysed by immunoblotting using anti-VDACl antibody.

For HeLa cell immunoprecipitation experiments, $8 \times 10^{6} \mathrm{HeLa}$ cells were transfected with the appropriate constructs. After $24 \mathrm{~h}$, cells were lysed in TNE buffer (10 mM Tris-HCl, $200 \mathrm{mM} \mathrm{NaCl}, 1 \mathrm{mM}$ EDTA (pH 7.4), $1 \mathrm{mM}$ $\beta$-glycerophosphate, $1 \mathrm{mM}$ orthovanadate, $0.1 \mathrm{mM}$ sodium pyrophosphate and

Figure 6 | Bcl-wav binds to zVDAC1 via its BH4 domain and controls mitochondrial calcium uptake. (a) Epifluorescence microscopy images of embryos at 75\% epiboly stained with Rhod-FF or Oregon Green Bapta-1 AM fluorescent dyes measuring mitochondrial and cytosolic [Ca $\left.{ }^{2+}\right]$, respectively. False colour was applied to visualize the differences in the fluorescence intensities reflecting $\left[\mathrm{Ca}^{2+}\right]$ changes. Dark blue: low $\left[\mathrm{Ca}^{2+}\right]$; yellow: high $\left[\mathrm{Ca}{ }^{2+}\right]$. Scale bar, $200 \mu \mathrm{m}$. (b) Histograms showing fold changes in the Rhod-FF/Oregon Green Bapta-1 AM fluorescence intensities of bc/wav morphants compared with controlMO (mean \pm s.d.; three independent experiments; ${ }^{\star} P<0.01$, Student's $t$-test). (c) Confocal stacks of controlMO versus bclwavMO embryos stained with Rhod-FF dye at 75\% epiboly. The enveloping cell layer (EVL), deep cell layer (DCL) and YSL of the embryo were analysed. Fluorescence intensities were visualized with false colour. Scale bar, $50 \mu \mathrm{m}$. (d) Representative response of transfected HeLa cells loaded with the mitochondrial $\mathrm{Ca}^{2}+$-sensitive dye Rhod-2. Mitochondrial $\mathrm{Ca}^{2+}$ uptake was stimulated with $5 \mu \mathrm{M}$ thapsigargin (black arrow). Scale bar, $60 \mathrm{~s}$. (e) Histograms depicting the quantitative response of the cells to $5 \mu \mathrm{M}$ thapsigargin (mean \pm s.d.; three independent experiments). (f) Analysis of the capacity of purified mitochondria from bclwavMO-, controlMO- or NADH ( $2 \mathrm{mM}$ )-treated embryos (75\% epiboly) to take up exogenous $\mathrm{Ca}^{2}+\left(20 \mu \mathrm{M} \mathrm{CaCl}_{2}\right)$. Calcium Green $5 \mathrm{~N}$ fluorescent dye was used to measure the decrease in extramitochondrial $\left[\mathrm{Ca}^{2+}\right]$, which directly reflects the mitochondrial uptake. Results are representative of two independent experiments. Scale bar, $60 \mathrm{~s}$. (g) Histogram showing the capacity of purified mitochondria to uptake exogenous $\mathrm{Ca}^{2+}$ as represented by a decrease in Oregon Green $5 \mathrm{~N}$ fluorescence intensity (FI)/second. Results are representative of two independent experiments. (h) Bcl-wav coimmunoprecipitates with endogenous zVDAC1 in purified mitochondria from zebrafish embryos. Immunoprecipitations were performed using homogeneity-purified mitochondrial extracts from embryos (30\% epiboly). Left lane (Mock), non-injected embryos; right lane (Flag-Bcl-wav), embryos injected with Flag-tagged bclwav transcripts. In both samples, two non-specific bands are revealed by the anti-VDAC1 antibody, the lower corresponding to the immunoglobulin light chain (black stars); however, in the sample prepared from embryos injected with Flag-tagged bclwav transcripts, a 34-kDa band corresponding to zVDAC1 can be detected (red arrow). (i) Bcl-wav interacts with endogenous VDAC1 via its BH4 domain in cellulo. Immunoprecipitation experiments were performed with protein extracts from transfected HeLa cells. Flag-Bcl-wav and Flag-Bcl-wav $\Delta T M$ were both able to interact with VDAC1 (upper red arrow) but not Flag- $\triangle \mathrm{BH} 4 \mathrm{BCl}$-wav; the FlagBH4 peptide domain also interacted with VDAC1 (lower red arrow). (j) Histograms showing the percentage of embryos with the bent notochord phenotype ( $26 \mathrm{hpf}$ ) among bclwav morphants co-injected with $\triangle B H 4 b c l w a v$ mRNA or $\triangle B H 4 b c l w a v$ mRNA alone (mean \pm s.d.; three independent experiments). (k) Histograms indicating the fold changes in Oregon Green Bapta- 1 fluorescence intensity of bclwav morphants injected or not with $\triangle B H 4 B c /$ wav mRNA compared with controlMO (mean \pm s.d.; three independent experiments; ${ }^{\star} P<0.01$, Student's $t$-test). Full scans of western blots can be found in Supplementary Fig. S10. 
a

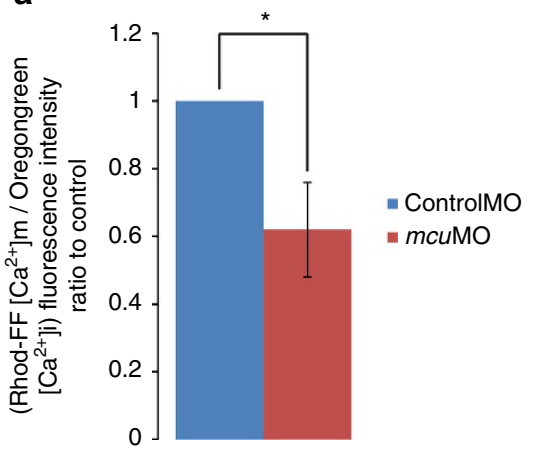

b
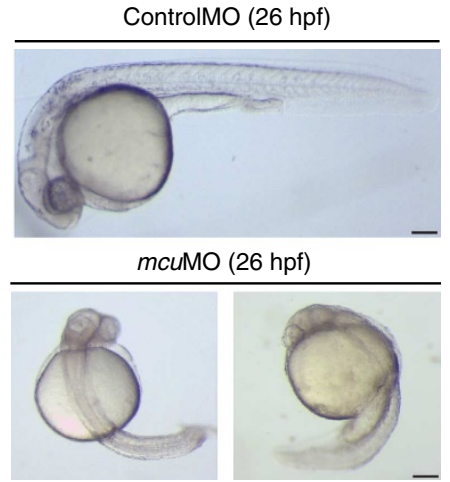

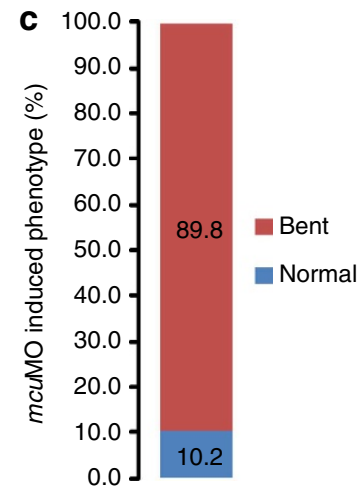

d

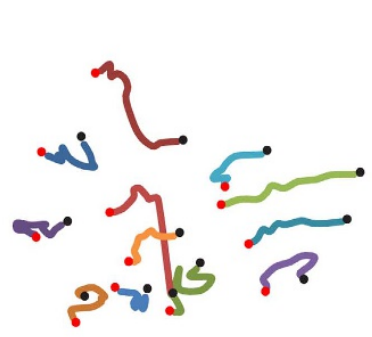

mcuMO
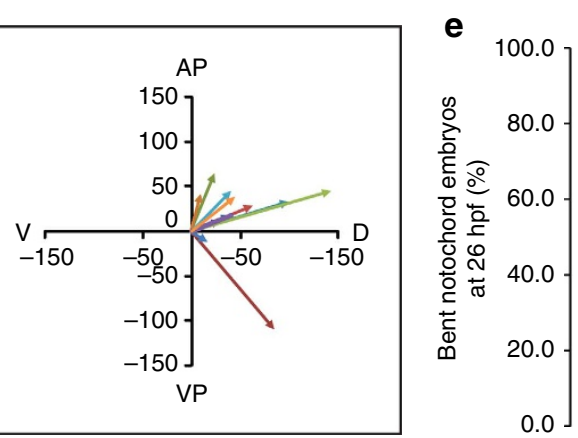

f
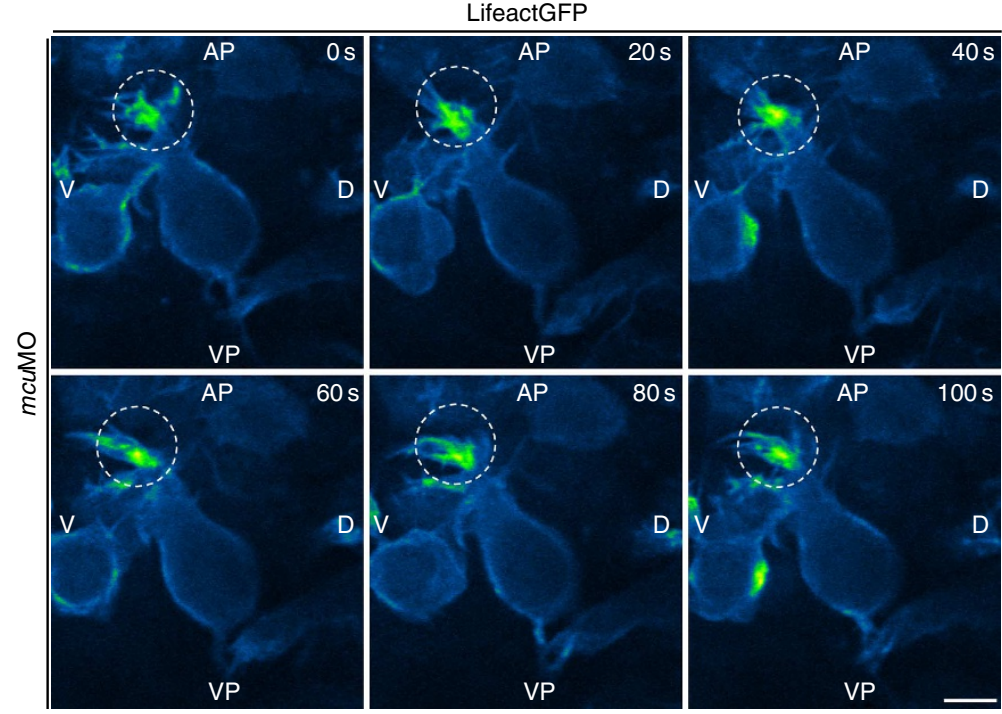

g

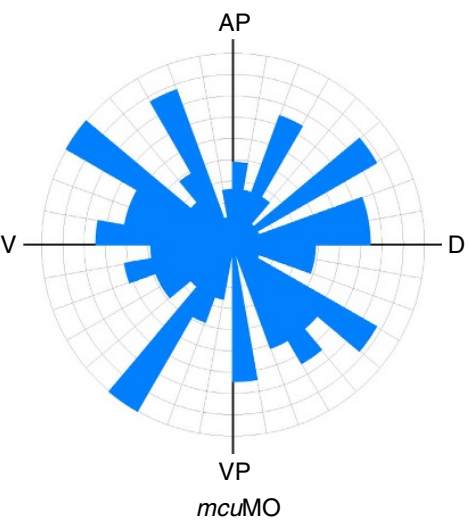

Figure 7 | Mcu knockdown phenocopies bclwav silencing. (a) Histograms indicating the fold changes in fluorescence intensity (Rhod-FF/Oregon Green Bapta-1 AM ratio) of mcu morphants compared with controlMO embryos (mean \pm s.d.; three independent experiments; ${ }^{\star} P<0.01$, Student's $t$-test). (b) Representative bright field images of mcuMO at $26 \mathrm{hpf}$ showing reduced anteroposterior axis formation and notochord deviation; these phenotypes are macroscopically identical to those observed in bclwav morphants. Scale bar, $200 \mu \mathrm{m}$. (c) Histogram showing the percentage of abnormal phenotypes (bent notochord phenotype) resulting from mcuMO injection at $26 \mathrm{hpf}$. (d) Cell paths and vector projections of single mesodermal cells observed during C\&E movements at the $75 \%$ epiboly stage in embryos injected with mcuMO, showing random cell migration. Vector projections are oriented as follows: $\mathrm{AP}$, animal pole; D, dorsal; $\mathrm{V}$, ventral; and VP, vegetal pole. (e) Histograms showing the percentage of bclwavMO embryos (blue), mcuMO embryos (red), bclwav and mcu morphants injected with Bapta-1 AM (green and purple, respectively) or Bapta-1 AM alone (light blue) at 26 hpf with the bent notochord phenotype (mean \pm s.d.; three independent experiments). (f) Confocal spinning disk analysis of F-actin dynamics of mcu morphants expressing lifeactgfp at the $75 \%$ epiboly stage, showing the location (white circles) and kinetics (seconds, bottom right corner) of F-actin polymerization. Image acquisition was performed on a single confocal section to visualize polymerized actin in lateral mesodermal cells. Scale bar, $10 \mu \mathrm{m}$. (g) Circular rose diagram of mcuMO embryos representing the orientation of actin protrusions. This diagram shows the frequency of protrusions with a given orientation. In mcuMO embryos, the direction of F-actin protrusions is randomized compared with control embryo. Diagrams are oriented as follows: AP, animal pole; $D$, dorsal; $\mathrm{V}$, ventral; and $\mathrm{VP}$, vegetal pole.

protease inhibitors). Extracts were precleared with protein G-Sepharose beads and subsequently incubated overnight with $3 \mu \mathrm{g}$ of anti-VDACl or $1 \mu \mathrm{g}$ of anti-HA antibodies. Extracts were then incubated for $3 \mathrm{~h}$ with protein $\mathrm{G}$-Sepharose beads and centrifuged for $5 \mathrm{~min}$ at 5,000 r.p.m. Pellets containing immunoprecipitated fractions were washed five times in TNE buffer and analysed by immunoblotting. The RhoA activation assay is described in Supplementary Methods. 
a

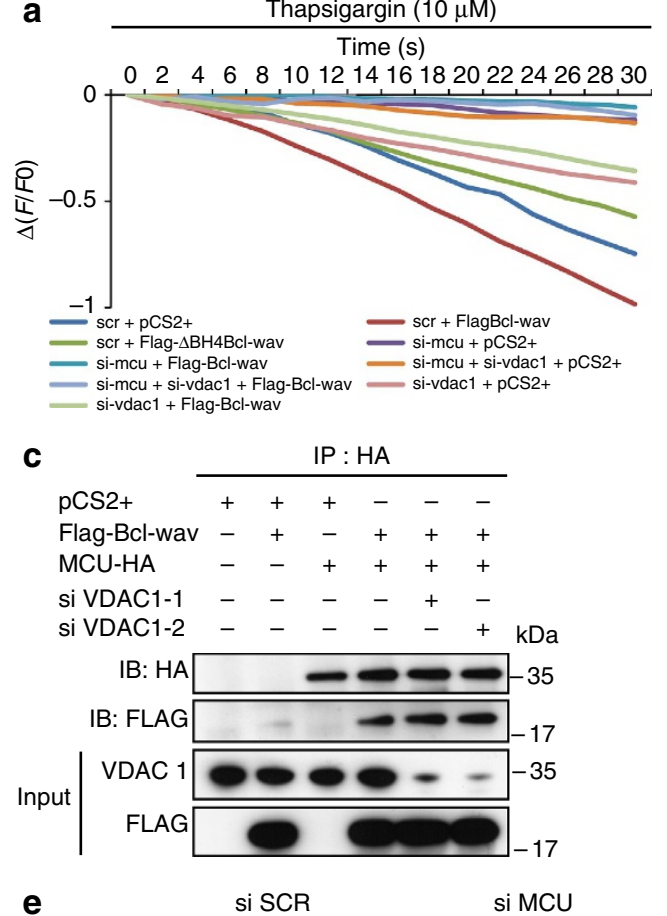

e

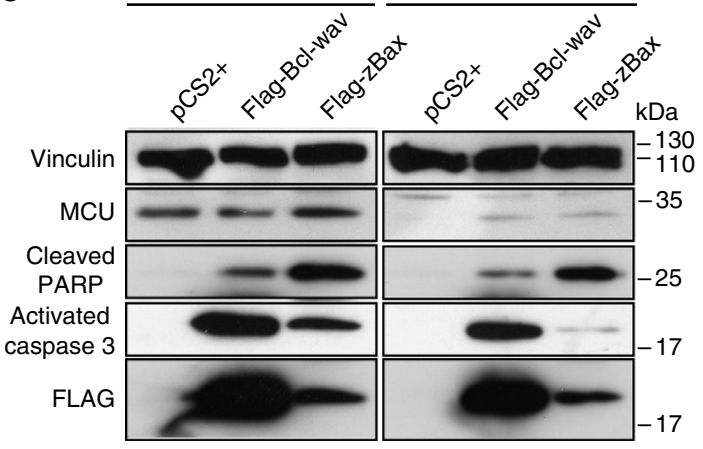

g

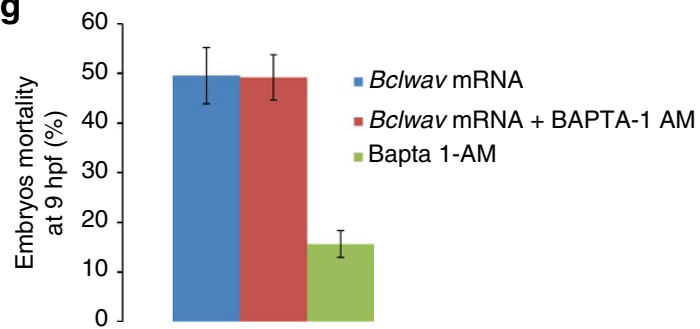

b

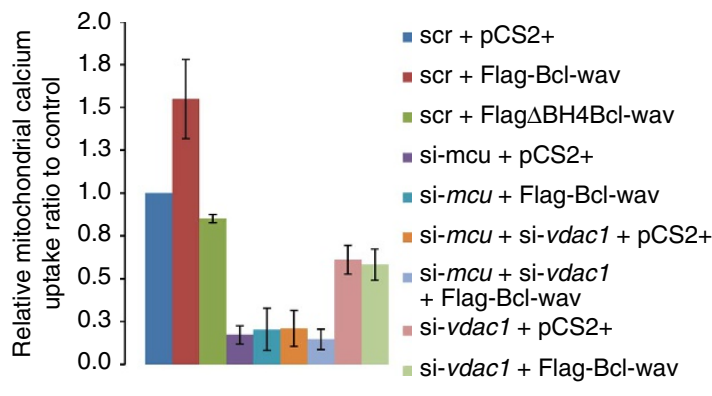

d
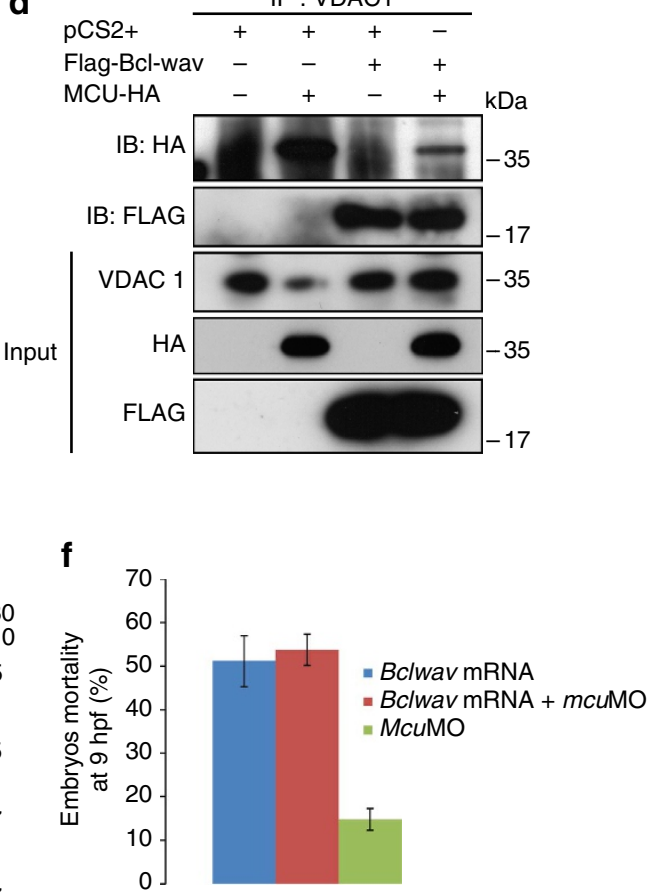

h

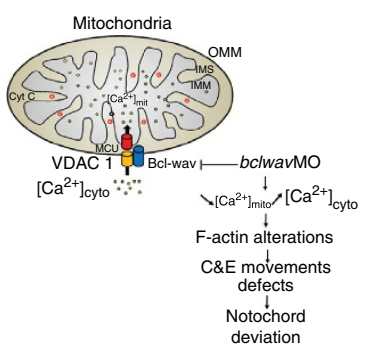

Figure 8 | The control of mitochondrial calcium by Bcl-wav, but not the induction of apoptosis, is VDAC1/MCU dependent. (a) Representative response of HeLa cells loaded with the intracellular $\mathrm{Ca}^{2+}$-sensitive dye FluoForte and stimulated with $10 \mu \mathrm{M}$ thapsigargin. The $\Delta \mathrm{F} / \mathrm{FO}$ decrease represents the mitochondrial calcium uptake measured during a 30-s period after the maximum of fluorescence intensity was reached. Cells were transfected with control scrambled (scr-), vdac1 (si-vdac1), mcu (si-mcu) or si-vdac1 + si-mcu small interfering RNAs in combination with empty vector (pCS2 +), pCS2 + Flag-Bclwav or pCS2 + Flag- $\triangle \mathrm{BH} 4 \mathrm{Bcl}$-wav. (b) Mitochondrial calcium uptake induced by Bcl-wav is VDAC1/MCU dependent. Histogram plot representing the relative calcium uptake during $30 \mathrm{~s}$ after the maximum fluorescence intensity was achieved, normalized to that in $\mathrm{scr}+\mathrm{pCS} 2+\mathrm{cells}$ (mean $\pm \mathrm{s}$.d.; three independent experiments). (c) Bcl-wav interacts with MCU-HA in cellulo. Co-immunoprecipitation was performed in protein extracts from HeLa cells (scr, si1- or si2-vdac1) transfected with pCS2 + Flag-Bcl-wav and pCS2 + MCU-HA using anti-HA antibody. Effiency of si-vdac1 was confirmed using an antiVDAC1 antibody. (d) Bcl-wav, VDAC1 and MCU form a protein complex at the mitochondria. Immunoprecipitation of endogenous VDAC1 was performed in HeLa cells expressing the empty vector (pCS2 +), Flag-Bcl-wav and/or MCU-HA. Both Flag-Bcl-wav and MCU-HA were detected in the co-transfected sample. (e) The pro-apoptotic activity of Bcl-wav is MCU independent. Caspase 3 is activated, and PARP is cleaved in both scr- and si-mcu HeLa cells transfected with $\mathrm{pCS} 2+$ Flag-Bcl-wav and $\mathrm{pCS} 2+$ Flag- $\triangle \mathrm{BH} 4 \mathrm{Bcl}$-wav; this result is not observed in control cells. Vinculin protein was used for calibration purposes. HeLa cells transfected with pCS2 + Flag-zBax were used as positive controls. (f) Overexpression of bclwav is lethal in mcu morphant embryos. The injection of bclwav mRNA into WT or mcuMO embryos at the one-cell stage induces early mortality during gastrulation (mean \pm s.d.; three independent experiments). (g) Histograms showing the percentage mortality of embryos injected with bclwav mRNA and Bapta-1 AM, alone or in combination (mean \pm s.d.; three independent experiments), at $9 \mathrm{hpf}$. (h) Model of Bcl-wav function during early zebrafish development. At mitochondria, Bcl-wav interacts with zVDAC1 and MCU to control $\mathrm{Ca}^{2+}$ entry. Bclwav knockdown increases free cytosolic $\mathrm{Ca}^{2+}$ levels, which may alter actin cytoskeletal dynamics, leading to changes in C\&E movements and subsequent notochord deviation. Full scans of western blots are provided in Supplementary Fig. S11. 
In vivo staining. For cytosolic and mitochondrial $\mathrm{Ca}^{2+}$ measurements, one-cell stage embryos were injected with $10 \mu \mathrm{M}$ Oregon Green 488 Bapta-1 AM or $10 \mu \mathrm{M}$ Rhod-FF, alone or in combination with RNA or morpholinos. Embryos were then analysed at 75\% epiboly using a Nikon TE300 fluorescence microscope.

For mitochondrial $\mathrm{Ca}^{2+}$ measurements in the YSL, enveloping layer and deep cell layer, one-cell stage was injected with $250 \mu \mathrm{M}$ Rhod-FF with controlMO or bclwavMO. Embryos were incubated at $28.5^{\circ} \mathrm{C}$ and subsequently analysed at $50-75 \%$ epiboly using a Zeiss 780 confocal microscope to specifically visualize each cell population. Images were acquired from 49 stacks of $4 \mu \mathrm{m}$ each per embryo.

For F-actin staining, embryos were fixed overnight in $4 \%$ paraformaldehyde at $4{ }^{\circ} \mathrm{C}$ and incubated with phalloidin ${ }^{44}$.

Active mitochondria at the $50 \%$ epiboly stage were stained by incubating embryos for $30 \mathrm{~min}$ in $500 \mathrm{nM}$ Mitotracker Red at $28.5^{\circ} \mathrm{C}$. Embryos were washed three times and visualized using a Nikon TE300 microscope.

Image acquisition was carried out with the same gain, amplification and exposure time for each experimental condition and corresponding control. Images were analysed using ImageJ software.

Mitochondrial calcium measurements in HeLa cells. Mitochondrial calcium in HeLa cells, transiently transfected with pCS2 +, pCS2 + Flag-Bcl-wav and pCS2 + Flag-zBcl-xL, was measured by monitoring Rhod-2 fluorescence in cells cultured in 96-well plates using a Mithras LB 940 multimode microplate reader (Berthold Technologies). Cells were loaded with $5 \mu \mathrm{M}$ Rhod-2 acetoxymethyl ester (Molecular Probes) in balanced salt solution (BBS) $(121 \mathrm{mM} \mathrm{NaCl}, 5.4 \mathrm{mM} \mathrm{KCl}$, $0.8 \mathrm{mM} \mathrm{MgCl}_{2}, 1.8 \mathrm{mM} \mathrm{CaCl}_{2}, 6 \mathrm{mM} \mathrm{NaHCO}_{3}, 5.5 \mathrm{mM} \mathrm{D}$-glucose and $25 \mathrm{mM}$ HEPES, pH 7.3) for $45 \mathrm{~min}$ at $37^{\circ} \mathrm{C}$. Following Rhod-2 loading, cells were incubated a further $30 \mathrm{~min}$ in fresh $\mathrm{Ca}^{2+}$-free $\mathrm{BBS}$ at room temperature for dye deesterification. Fluorescence values were obtained by excitation at $550 \mathrm{~nm}$, and emission signal was collected at $580 \mathrm{~nm}$ every $400 \mathrm{~ms}$. Thapsigargin in $\mathrm{Ca}^{2+}$-free BBS was injected in each well after $15 \mathrm{~s}$ of measurement at a final concentration of $5 \mu \mathrm{M}$.

Extramitochondrial free $\mathrm{Ca}^{2+}$ was monitored in scr-, si- $m c u$ and/or si-vdacl HeLa cells transiently transfected with pCS2 + , Flag-Bcl-wav or Flag- $\Delta$ BH4Bclwav using the FluoForte probe (Enzo). Cells were loaded with $5 \mu \mathrm{M}$ FluoForte for $1 \mathrm{~h}$ at $37^{\circ} \mathrm{C}$ in fresh $\mathrm{Ca}^{2+}$-free BBS. Fluoforte fluorescence was monitored every $2 \mathrm{~s}$ using a Zeiss 780 confocal microscope after thapsigargin injection (10 $\mu \mathrm{M}$ final). Relative mitochondrial calcium uptake was calculated by computing linear fits for $30 \mathrm{~s}$ after the maximum fluorescence intensity was reached.

\section{Calcium uptake capacity of isolated zebrafish mitochondria. Extra-} mitochondrial free $\mathrm{Ca}^{2}+$ was monitored in the presence of isolated mitochondria using Oregon Green $5 \mathrm{~N}$ hexapotassium salt. Purified mitochondria from $75 \%$ epiboly embryos were resuspended in $\mathrm{KCl}$ medium $\left(125 \mathrm{mM} \mathrm{KCl}, 2 \mathrm{mM} \mathrm{K}_{2} \mathrm{HPO}_{4}\right.$, $1 \mathrm{mM} \mathrm{MgCl}{ }_{2}$ and $20 \mathrm{mM}$ HEPES, pH 7) containing $1 \mu \mathrm{M}$ Oregon Green $5 \mathrm{~N}$ (Molecular Probes) and supplemented with $5 \mathrm{mM}$ glutamate and $5 \mathrm{mM}$ malate. Oregon Green 5N fluorescence was measured using a Mithras LB 940 multimode microplate reader (Berthold Technologies) with excitation at $485 \mathrm{~nm}$ and emission at $510 \mathrm{~nm} . \mathrm{CaCl}_{2}$ (in supplemented $\mathrm{KCl}$ medium) was injected after $30 \mathrm{~s}$ of measurement at a final concentration of $20 \mu \mathrm{M}$.

Time-lapse microscopy. For cell tracing experiments, embryos co-injected with controlMO or bclwavMO and lifeactgfp mRNA were manually dechorionated at the shield stage and incorporated into $0.5 \%$ low-melting point agarose. Before solidification, embryos were orientated in the dorsoventral plane to permit observation of the lateral mesoderm. Embryos were then observed using a Leica DMI4000 inverted microscope equipped with a $28^{\circ} \mathrm{C}$ observation chamber, an EMCCD camera (Photometrics Quantem 512) and a $\times 40$ oil immersion objective. Time-lapse images were acquired every $20 \mathrm{~s}$, and total acquisition time was $20 \mathrm{~min}$. Image analyses were performed using ImageJ.

Transplantation. Embryos expressing $\beta$-actin-GFP were used as control donors, whereas bclwavMO was co-injected with rhodamine. Cells from control and morphant donors were removed at 50\% epiboly and transplanted into the shield of the same WT receiver embryo at shield stage. Transplantation was verified under a fluorescence microscope. Cell migration in receiver embryos was analysed at $90 \%$ epiboly.

Statistical analyses. Error bars displayed on graphs represent the means \pm s.d. of three independent experiments. Statistical significance was analysed using Student's $t$-test. $P<0.01$ was considered significant.

Co-localization percentage. Co-localization percentage between Flag-Bcl-wav and mitochondria labelled with MitoTracker was calculated with Zen software. Details regarding subcellular localization experiments are provided in Supplementary Methods.

\section{References}

1. Youle, R. J. \& Strasser, A. The BCL-2 protein family: opposing activities that mediate cell death. Nature reviews. Mol. Cell Biol. 9, 47-59 (2008).

2. Wang, C. \& Youle, R. J. The role of mitochondria in apoptosis*. Annu. Rev. Genet. 43, 95-118 (2009)

3. Berridge, M. J., Bootman, M. D. \& Roderick, H. L. Calcium signalling: dynamics, homeostasis and remodelling. Nat. Rev. Mol. Cell Biol. 4, 517-529 (2003).

4. Rizzuto, R. et al. $\mathrm{Ca}(2+)$ transfer from the ER to mitochondria: when, how and why. Biochim. Biophys. Acta 1787, 1342-1351 (2009).

5. Rapizzi, E. et al. Recombinant expression of the voltage-dependent anion channel enhances the transfer of $\mathrm{Ca} 2+$ microdomains to mitochondria. J. Cell Biol. 159, 613-624 (2002).

6. De Stefani, D., Raffaello, A., Teardo, E., Szabo, I. \& Rizzuto, R. A fortykilodalton protein of the inner membrane is the mitochondrial calcium uniporter. Nature 476, 336-340 (2011).

7. Baughman, J. M. et al. Integrative genomics identifies $\mathrm{MCU}$ as an essential component of the mitochondrial calcium uniporter. Nature 476, 341-345 (2011).

8. Bonneau, B., Prudent, J., Popgeorgiev, N. \& Gillet, G. Non-apoptotic roles of Bcl-2 family: the calcium connection. Biochim. Biophys. Acta 1833, 1755-1765 (2013).

9. Eimon, P. M. \& Ashkenazi, A. The zebrafish as a model organism for the study of apoptosis. Apoptosis 15, 331-349 (2010).

10. Sepich, D. S., Usmani, M., Pawlicki, S. \& Solnica-Krezel, L. Wnt/PCP signaling controls intracellular position of MTOCs during gastrulation convergence and extension movements. Development (Cambridge, England) 138, 543-552 (2011).

11. Kratz, E. et al. Functional characterization of the Bcl-2 gene family in the zebrafish. Cell Death Differ. 13, 1631-1640 (2006).

12. Webb, S. E. \& Miller, A. L. Calcium signalling during embryonic development. Nat. Rev. Mol. Cell Biol. 4, 539-551 (2003).

13. Arnaud, E. et al. The zebrafish bcl-2 homologue Nrz controls development during somitogenesis and gastrulation via apoptosis-dependent and -independent mechanisms. Cell Death Differ. 13, 1128-1137 (2006)

14. Popgeorgiev, N. et al. The apoptotic regulator Nrz controls cytoskeletal dynamics via the regulation of $\mathrm{Ca}(2+)$ trafficking in the zebrafish blastula. Dev. Cell 20, 663-676 (2011).

15. Aouacheria, A., Rech de Laval, V., Deléage, G. \& Combet, C. Characterization of the Bcl-2 family using structure-aided HMM Framework. JOBIM 79-80 (2010).

16. Kane, D. A. \& Kimmel, C. B. The zebrafish midblastula transition. Development 119, 447-456 (1993).

17. Gerety, S. S. \& Wilkinson, D. G. Morpholino artifacts provide pitfalls and reveal a novel role for pro-apoptotic genes in hindbrain boundary development. Dev. Biol. 350, 279-289 (2011).

18. Tan, W. \& Colombini, M. VDAC closure increases calcium ion flux. Biochim Biophys. Acta 1768, 2510-2515 (2007).

19. De Stefani, D. et al. VDAC1 selectively transfers apoptotic $\mathrm{Ca} 2+$ signals to mitochondria. Cell Death Differ. 19, 267-273 (2012).

20. Roy, S. S. et al. Bad targets the permeability transition pore independent of Bax or Bak to switch between Ca2 +-dependent cell survival and death. Mol. Cell 33, 377-388 (2009)

21. Shimizu, S., Konishi, A., Kodama, T. \& Tsujimoto, Y. BH4 domain of antiapoptotic Bcl-2 family members closes voltage-dependent anion channel and inhibits apoptotic mitochondrial changes and cell death. Proc. Natl Acad Sci. USA 97, 3100-3105 (2000).

22. Vander Heiden, M. G. et al. Bcl-xL promotes the open configuration of the voltage-dependent anion channel and metabolite passage through the outer mitochondrial membrane. J. Biol. Chem. 276, 19414-19419 (2001).

23. Vander Heiden, M. G., Chandel, N. S., Schumacker, P. T. \& Thompson, C. B. $\mathrm{Bcl}-\mathrm{xL}$ prevents cell death following growth factor withdrawal by facilitating mitochondrial ATP/ADP exchange. Mol. Cell 3, 159-167 (1999).

24. Wallingford, J. B., Ewald, A. J., Harland, R. M. \& Fraser, S. E. Calcium signaling during convergent extension in Xenopus. Curr. Biol. 11, 652-661 (2001).

25. Markova, O. \& Lenne, P. F. Calcium signaling in developing embryos: Focus on the regulation of cell shape changes and collective movements. Semin. Cell Dev. Biol. 23, 298-307 (2012).

26. Gilland, E., Miller, A. L., Karplus, E., Baker, R. \& Webb, S. E. Imaging of multicellular large-scale rhythmic calcium waves during zebrafish gastrulation. Proc. Natl Acad. Sci. USA 96, 157-161 (1999).

27. Freisinger, C. M., Fisher, R. A. \& Slusarski, D. C. Regulator of g protein signaling 3 modulates wnt5b calcium dynamics and somite patterning. PLoS Genet. 6, 1001020 (2010).

28. Guillemin, Y. et al. Oocytes and early embryos selectively express the survival factor BCL2L10. J. Mol. Med. 87, 923-940 (2009).

29. Guillemin, Y., Cornut-Thibaut, A., Gillet, G., Penin, F. \& Aouacheria, A. Characterization of unique signature sequences in the divergent maternal protein Bcl2110. Mol. Biol. Evol. 28, 3271-3283 (2011). 
30. Rinkenberger, J. L., Horning, S., Klocke, B., Roth, K. \& Korsmeyer, S. J. Mcl-1 deficiency results in peri-implantation embryonic lethality. Genes Dev. 14, 23-27 (2000).

31. Aouacheria, A., Brunet, F. \& Gouy, M. Phylogenomics of life-or-death switches in multicellular animals: Bcl-2, BH3-Only, and BNip families of apoptotic regulators. Mol. Biol. Evol. 22, 2395-2416 (2005).

32. Roy, S. S., Ehrlich, A. M., Craigen, W. J. \& Hajnoczky, G. VDAC2 is required for truncated BID-induced mitochondrial apoptosis by recruiting BAK to the mitochondria. EMBO Rep. 10, 1341-1347 (2009).

33. Tsujimoto, Y. \& Shimizu, S. VDAC regulation by the Bcl-2 family of proteins. Cell Death Differ. 7, 1174-1181 (2000).

34. Tada, M., Concha, M. L. \& Heisenberg, C. P. Non-canonical Wnt signalling and regulation of gastrulation movements. Semin. Cell Dev. Biol. 13, 251-260 (2002).

35. Zhu, S., Liu, L., Korzh, V., Gong, Z. \& Low, B. C. RhoA acts downstream of Wnt5 and Wnt11 to regulate convergence and extension movements by involving effectors Rho kinase and diaphanous: use of zebrafish as an in vivo model for GTPase signaling. Cell Signal 18, 359-372 (2006).

36. Kardash, E. et al. A role for Rho GTPases and cell-cell adhesion in single-cell motility in vivo. Nat. Cell Biol. 12, 47-53 (2010).

37. Sheldahl, L. C. et al. Dishevelled activates Ca2 + flux, PKC, and CamKII in vertebrate embryos. J. Cell Biol. 161, 769-777 (2003).

38. Kohn, A. D. \& Moon, R. T. Wnt and calcium signaling: beta-cateninindependent pathways. Cell Calcium 38, 439-446 (2005).

39. Webb, S. E. \& Miller, A. L. Ca2 + signalling and early embryonic patterning during zebrafish development. Clin. Exp. Pharmacol. Physiol. 34, 897-904 (2007).

40. Yin, C. \& Solnica-Krezel, L. Convergence and extension movements mediate the specification and fate maintenance of zebrafish slow muscle precursors. Dev. Biol. 304, 141-155 (2007).

41. Moradi-Ameli, M., Lorca, T., Ficheux, D., di Pietro, A. \& Gillet, G. Interaction between the antiapoptotic protein $\mathrm{Nr}-13$ and cytochrome $\mathrm{c}$. Antagonistic effect of the BH3 domain of Bax. Biochemistry 41, 8540-8550 (2002).

42. Gillet, G., Guerin, M., Trembleau, A. \& Brun, G. A Bcl-2-related gene is activated in avian cells transformed by the Rous sarcoma virus. EMBO J. 14 1372-1381 (1995).

43. Thisse, C. \& Thisse, B. High-resolution in situ hybridization to whole-mount zebrafish embryos. Nat. Protoc. 3, 59-69 (2008).
44. Koppen, M., Fernandez, B. G., Carvalho, L., Jacinto, A. \& Heisenberg, C. P. Coordinated cell-shape changes control epithelial movement in zebrafish and Drosophila. Development (Cambridge, England) 133, 2671-2681 (2006).

\section{Acknowledgements}

We wish to thank the technical staff of the zebrafish and cell imaging facilities at Ecole Normale Supérieure de Lyon (Lyon Biosciences UMS 3444), and we thank Alexander Wood for reading the manuscript. This work was supported by the Association pour la Recherche sur le Cancer (ARC) and the Ligue Nationale contre le Cancer (Comite de la Drôme). J.P. is supported by the Région Rhône Alpes and the Fondation pour la recherché Médicale. N.P. and B.B. are supported by the ARC and the Ministère de la Recherche.

\section{Author contributions}

J.P., N.P. and B.B. performed all experiments not otherwise attributed to other authors. J.P. and J.T. produced Bcl-wav protein and antibodies. J.P., J.L. and P.G. measured the oxygen consumption rate. S.M. performed the yeast experiments. B.B. and C.H. performed cell transplantation experiments. R.G. contributed to western blotting and co-immunoprecipitation assays. P.H. and R.R. contributed to the understanding of the $b c l w a v \mathrm{MO}$ phenotype. A.A. identified the bclwav sequence. J.P., N.P., B.B. and G.G. wrote the paper.

\section{Additional information}

Accession codes: Sequence data have been deposited in GenBank/EMBL/DDBJ under accession number GU350411.1.

Supplementary Information accompanies this paper at http://www.nature.com/ naturecommunications

Competing financial interests: The authors declare no competing financial interests.

Reprints and permission information is available online at http://npg.nature.com/ reprintsandpermissions/

How to cite this article: Prudent, J. et al. Bcl-wav and the mitochondrial calcium uniporter drive gastrula morphogenesis in zebrafish. Nat. Commun. 4:2330 doi: $10.1038 /$ ncomms3330 (2013). 